\title{
DIAGRAM ALGEBRAS, DOMINANCE TRIANGULARITY, AND SKEW CELL MODULES
}

\author{
CHRISTOPHER BOWMAN, JOHN ENYANG, AND FREDERICK GOODMAN
}

\begin{abstract}
We present an abstract framework for the axiomatic study of diagram algebras. Algebras that fit this framework possess analogues of both the Murphy and seminormal bases of the Hecke algebras of the symmetric groups. We show that the transition matrix between these bases is dominance unitriangular. We construct analogues of the skew Specht modules in this setting. This allows us to propose a natural tableaux theoretic framework in which to study the infamous Kronecker problem.
\end{abstract}

\section{INTRODUCTION}

The purpose of this article is to develop an Okounkov-Vershik-style framework in which to study towers of diagram algebras $\left(A_{r}\right)_{r \geqslant 0}$ over an integral domain, $R$, and its field of fractions, $\mathbb{F}$. The diagram algebras that fit into our framework include group algebras of the symmetric groups and their Hecke algebras, the Brauer and BMW algebras, walled Brauer algebras, Jones-Temperley-Lieb algebras, as well as centralizer algebras for the general linear, orthogonal, and symplectic groups acting on tensor spaces.

Following [4], we observe that algebras fitting into our framework possess analogues of both the Murphy and seminormal bases of the Hecke algebras of the symmetric groups. We prove that the transition matrix between these bases is dominance unitriangular. In case the algebras have Jucys-Murphy elements, we prove that these elements act diagonally on the seminormal basis and triangularly with respect to dominance order on the Murphy basis. For the Hecke algebras of symmetric groups, this provides a new and very simple proof of dominance triangularity of the Jucys-Murphy elements (see [14, Theorem 4.6], [11, Theorem 3.32 and Proposition 3.35]). For other diagram algebras such as the Brauer algebras, walled Brauer algebras, BMW algebras or partition algebras, dominance triangularity is a new result. Dominance triangularity is an extremely useful structural result, which has already found two distinct applications which we highlight below.

There is a deeper structure of the Murphy cellular bases of diagram algebras which underlies dominance triangularity. Elements of the Murphy

1991 Mathematics Subject Classification. 20G05, 05E10.

Key words and phrases. Cellular algebras, diagram algebras. 
basis can be written using an ordered product of certain "branching factors". There exist both "down" and "up" branching factors and a compatibility relation between them. Using this compatibility relation, one obtains a certain factorizability property of the Murphy basis elements. The compatibility and factorizability properties lead to our dominance triangularity results, but also to strong results about restrictions of cell modules, the construction of skew cell modules, and in [3] to a construction of cellular basis for quotients of diagram algebras. The compatibility and factorizability properties were already observed in [4], but they are first exploited systematically in this paper and in [3].

The results of this paper play a crucial role in [3], where we construct new integral Murphy-type cellular bases of the Brauer algebras which decompose into bases for the kernels and images of these algebras acting on tensor space. This construction thus provides simultaneously an integral cellular basis of the centralizer algebra, and a new version of the second fundamental theorem of invariant theory. All of these results are compatible with reduction from $\mathbb{Z}$ to an arbitrary field (characteristic 2 is excluded in the orthogonal case).

Given two fixed points $\lambda$ and $\nu$ in the $s$ th and $r$ th levels of the branching graph, we provide an explicit construction of an associated skew cell module $\Delta_{r-s}(\nu \backslash \lambda)$. We show that these skew cell modules possess integral bases indexed by skew tableaux (paths between the two fixed vertices in the graph) exactly as in the classical case of the symmetric group.

In the case of the partition algebra, these skew modules provide a new setting in which to study the infamous Kronecker problem. In an upcoming paper [1], the first two authors and Maud De Visscher use these skew modules to provide a uniform combinatorial interpretation for one of the largest sets of Kronecker coefficients considered to date (the Littlewood-Richardson coefficients and the Kronecker coefficients labelled by two 2-line partitions are covered as important examples).

\section{Diagram ALgebras}

For the remainder of the paper, we shall let $R$ be an integral domain with field of fractions $\mathbb{F}$. In this section, we shall define diagram algebras and recall the construction of their Murphy bases, following [4]. We first recall the definition of a cellular algebra, as in [9].

\subsection{Cellular algebras.}

Definition 2.1. Let $R$ be an integral domain. A cellular algebra is a tuple $(A, *, \widehat{A}, \unrhd, \operatorname{Std}(\cdot), \mathscr{A})$ where

(1) $A$ is a unital $R$-algebra and $*: A \rightarrow A$ is an algebra involution, that is, an $R$-linear anti-automorphism of $A$ such that $\left(x^{*}\right)^{*}=x$ for $x \in A$;

(2) $(\widehat{A}, \unrhd)$ is a finite partially ordered set, and for each $\lambda \in \widehat{A}, \operatorname{Std}(\lambda)$ is a finite indexing set;

(3) The set $\mathscr{A}=\left\{c_{\mathrm{st}}^{\lambda} \mid \lambda \in \widehat{A}\right.$ and $\left.\mathrm{s}, \mathrm{t} \in \operatorname{Std}(\lambda)\right\}$ is an $R$-basis for $A$.

Let $A^{\triangleright \lambda}$ denote the $R$-module with basis $\left\{c_{\mathrm{st}}^{\mu} \mid \mu \triangleright \lambda\right.$ and $\left.\mathrm{s}, \mathrm{t} \in \operatorname{Std}(\mu)\right\}$. 
(4) The following two conditions hold for the basis $\mathscr{A}$.

(a) Given $\lambda \in \widehat{A}, \mathrm{t} \in \operatorname{Std}(\lambda)$, and $a \in A$, there exist coefficients $r(a ; \mathrm{t}, \mathrm{v}) \in R$, for $\mathrm{v} \in \operatorname{Std}(\lambda)$, such that, for all $\mathrm{s} \in \operatorname{Std}(\lambda)$,

$$
c_{\mathrm{st}}^{\lambda} a \equiv \sum_{\mathrm{v} \in \operatorname{Std}(\lambda)} r(a ; \mathrm{t}, \mathrm{v}) c_{\mathrm{sv}}^{\lambda} \bmod A^{\triangleright \lambda},
$$

(b) If $\lambda \in \widehat{A}$ and $\mathrm{s}, \mathrm{t} \in \operatorname{Std}(\lambda)$, then $\left(c_{\mathrm{st}}^{\lambda}\right)^{*} \equiv\left(c_{\mathrm{ts}}^{\lambda}\right) \bmod A^{\triangleright \lambda}$.

The tuple $(A, *, \widehat{A}, \unrhd, \operatorname{Std}(\cdot), \mathscr{A})$ is a cell datum for $A$. The basis $\mathscr{A}$ is called a cellular basis of $A$.

If $A$ is a cellular algebra over $R$, and $R \rightarrow S$ is a homomorphism of integral domains, then the specialization $A^{S}=A \otimes_{R} S$ is a cellular algebra over $S$, with cellular basis

$$
\mathscr{A}^{S}=\left\{c_{\mathrm{st}}^{\lambda} \otimes 1_{S} \mid \lambda \in \widehat{A}, \text { and } \mathrm{s}, \mathrm{t} \in \operatorname{Std}(\lambda)\right\} .
$$

In particular, $A^{\mathbb{F}}$ is a cellular algebra. Since the map $a \mapsto a \otimes 1_{\mathbb{F}}$ is injective, we regard $A$ as contained in $A^{\mathbb{F}}$ and we identify $a \in A$ with $a \otimes 1_{\mathbb{F}} \in A^{\mathbb{F}}$.

An order ideal $\Gamma \subset \widehat{A}$ is a subset with the property that if $\lambda \in \Gamma$ and $\mu \triangleq \lambda$, then $\mu \in \Gamma$. It follows from the axioms of a cellular algebra that for any order ideal $\Gamma$ in $\widehat{A}$,

$$
A^{\Gamma}=\operatorname{Span}\left\{c_{\mathrm{st}}^{\lambda} \mid \lambda \in \Gamma, \mathrm{s}, \mathrm{t} \in \operatorname{Std}(\lambda)\right\}
$$

is an involution-invariant two sided ideal of $A$. In particular $A^{\triangleright \lambda}$ and

$$
A^{\unrhd \lambda}=\operatorname{Span}\left\{c_{\mathrm{st}}^{\mu} \mid \mu \in \hat{A}, \mathfrak{S}, \mathrm{t} \in \operatorname{Std}(\mu) \text { and } \mu \unrhd \lambda\right\}
$$

are involution-invariant two sided ideals.

Definition 2.2. Let $A$ be a cellular algebra over $R$ and $\lambda \in \hat{A}$. The cell module $\Delta(\lambda)$ is the right $A$-module defined as follows. As an $R$-module, $\Delta(\lambda)$ is free with basis indexed by $\operatorname{Std}(\lambda)$, say $\left\{c_{\mathrm{t}}^{\lambda} \mid \mathrm{t} \in \operatorname{Std}(\lambda)\right\}$. The right $A$-action is given by

$$
c_{\mathrm{t}}^{\lambda} a=\sum_{\mathrm{v} \in \hat{A}^{\lambda}} r(a ; \mathrm{t}, \mathrm{v}) c_{\mathrm{v}}^{\lambda},
$$

where the coefficients $r(a ; \mathrm{t}, \mathrm{v})$ are those of Equation (1).

Thus, for any $\mathrm{s} \in \widehat{A}^{\lambda}$,

$$
\operatorname{Span}\left\{c_{\mathrm{st}}^{\lambda}+A^{\triangleright \lambda} \mid \mathrm{t} \in \operatorname{Std}(\lambda)\right\} \subseteq A^{\unrhd \lambda} / A^{\triangleright \lambda}
$$

is a model for the cell module $\Delta(\lambda)$. When we need to emphasize the algebra or the ground ring, we may write $\Delta_{A}(\lambda)$ or $\Delta^{R}(\lambda)$. Note that $\Delta^{\mathbb{F}}(\lambda)=\Delta(\lambda) \otimes_{R} \mathbb{F}$ is the cell module for $A^{\mathbb{F}}$ corresponding to $\lambda$.

If $A$ is an $R$-algebra with involution $*$, then $*$ induces functors $M \rightarrow M^{*}$ interchanging left and right $A-$ modules, and taking $A-A$ bimodules to $A-A$ bimodules. We identify $M^{* *}$ with $M$ via $x^{* *} \mapsto x$ and for modules ${ }_{A} M$ and $N_{A}$ we have $\left(M \otimes_{R} N\right)^{*} \cong N^{*} \otimes_{R} M^{*}$, as $A-A$ bimodules, with the isomorphism determined by $(m \otimes n)^{*} \mapsto n^{*} \otimes m^{*}$. For a right $A$-module $M_{A}$, using both of these isomorphisms, we identify $\left(M^{*} \otimes M\right)^{*}$ with $M^{*} \otimes M^{* *}=$ 
$M^{*} \otimes M$, via $\left(x^{*} \otimes y\right)^{*} \mapsto y^{*} \otimes x$. Now we apply these observations with $A$ a cellular algebra and $\Delta(\lambda)$ a cell module. The assignment

$$
\alpha_{\lambda}: c_{\mathrm{st}}^{\lambda}+A^{\triangleright \lambda} \mapsto\left(c_{\mathrm{s}}^{\lambda}\right)^{*} \otimes\left(c_{\mathrm{t}}^{\lambda}\right)
$$

determines an $A-A$ bimodule isomorphism from $A^{\unrhd \lambda} / \mathrm{A}^{\triangleright \lambda}$ to $(\Delta(\lambda))^{*} \otimes_{R}$ $\Delta(\lambda)$. Moreover, we have $* \circ \alpha_{\lambda}=\alpha_{\lambda} \circ *$, which reflects the cellular algebra axiom $\left(c_{\mathrm{st}}^{\lambda}\right)^{*} \equiv c_{\mathrm{ts}}^{\lambda} \bmod A^{\triangleright \lambda}$.

A certain bilinear form on the cell modules plays an essential role in the theory of cellular algebras. Let $A$ be a cellular algebra over $R$ and let $\lambda \in \widehat{A}$. The cell module $\Delta(\lambda)$ can be regarded as an $A / A^{\triangleright \lambda}$ module. For $x, y, z \in \Delta(\lambda)$, it follows from the the definition of the cell module and the map $\alpha_{\lambda}$ that $x \alpha_{\lambda}^{-1}\left(y^{*} \otimes z\right) \in R z$. Define $\langle x, y\rangle$ by

$$
x \alpha_{\lambda}^{-1}\left(y^{*} \otimes z\right)=\langle x, y\rangle z .
$$

Then $\langle x, y\rangle$ is $R$-linear in each variable and we have $\langle x a, y\rangle=\left\langle x, y a^{*}\right\rangle$ for $x, y \in \Delta(\lambda)$ and $a \in A$. Note that

$$
c_{\mathrm{st}}^{\lambda} c_{\mathrm{uv}}^{\lambda}=\left\langle c_{\mathrm{t}}^{\lambda}, c_{\mathrm{u}}^{\lambda}\right\rangle c_{\mathrm{sv}}^{\lambda},
$$

which is the customary definition of the bilinear form.

Definition 2.3 ([5]). A cellular algebra, $A$, is said to be cyclic cellular if every cell module is cyclic as an $A$-module.

If $A$ is cyclic cellular, $\lambda \in \widehat{A}$, and $\delta(\lambda)$ is a generator of the cell module $\Delta(\lambda)$, let $m_{\lambda}$ be a lift in $A^{\unrhd \lambda}$ of $\alpha_{\lambda}^{-1}\left(\delta(\lambda)^{*} \otimes \delta(\lambda)\right)$.

Lemma 2.4. The element $m_{\lambda}$ has the following properties:

(1) $m_{\lambda} \equiv m_{\lambda}^{*} \bmod A^{\triangleright \lambda}$.

(2) $A^{\unrhd \lambda}=A m_{\lambda} A+A^{\triangleright \lambda}$.

(3) $\left(m_{\lambda} A+A^{\triangleright \lambda}\right) / A^{\triangleright \lambda} \cong \Delta(\lambda)$, as right $A$-modules.

Proof. Lemma 2.5 in [5].

In examples of interest to us, we can always choose $m_{\lambda}$ to satisfy $m_{\lambda}^{*}=m_{\lambda}$ (and moreover, $m_{\lambda}$ is given explicitly).

2.2. Sequences of diagram algebras. Here and in the remainder of the paper, we will consider an increasing sequence $\left(A_{r}\right)_{r \geqslant 0}$ of cellular algebras over an integral domain $R$ with field of fractions $\mathbb{F}$. We assume that all the inclusions are unital and that the involutions are consistent; that is the involution on $A_{r+1}$, restricted to $A_{r}$, agrees with the involution on $A_{r}$. We will establish a list of assumptions (D1)-(D6). For convenience, we call an increasing sequence of cellular algebras satisfying these assumptions a sequence of diagram algebras.

Let $\left(\widehat{A}_{r}, \triangleq\right)$ denote the partially ordered set in the cell datum for $A_{r}$. For $\lambda \in \widehat{A}_{r}$, let $\Delta_{r}(\lambda)$ denote the corresponding cell module. If $S$ is an integral domain with a unital homomorphism $R \rightarrow S$, write $A_{r}^{S}=A_{r} \otimes_{R} S$ and $\Delta_{r}^{S}(\lambda)$ for $\Delta_{r}(\lambda) \otimes_{R} S$. In particular, write $A_{r}^{\mathbb{F}}=A_{r} \otimes_{R} \mathbb{F}$ and $\Delta_{r}^{\mathbb{F}}(\lambda)$ for $\Delta_{r}(\lambda) \otimes_{R} \mathbb{F}$. 
Definition 2.5. Let $A$ be a cellular algebra over $R$. If $M$ is a right $A_{-}$ module, a cell-filtration of $M$ is a filtration by right $A$-modules

$$
\{0\}=M_{0} \subseteq M_{1} \subseteq \cdots \subseteq M_{s}=M,
$$

such that $M_{i} / M_{i-1} \cong \Delta\left(\lambda^{(i)}\right)$ for some $\lambda^{(i)} \in \widehat{A}$. We say that the filtration is order preserving if $\lambda^{(i)} \triangleright \lambda^{(i+1)}$ in $\widehat{A}$ for all $i \geqslant 1$.

Definition $2.6([6,7])$. Let $\left(A_{r}\right)_{r \geqslant 0}$ be an increasing sequence of cellular algebras over an integral domain $R$.

(1) The tower $\left(A_{r}\right)_{r \geqslant 0}$ is restriction-coherent if for each $r \geqslant 0$ and each $\mu \in$

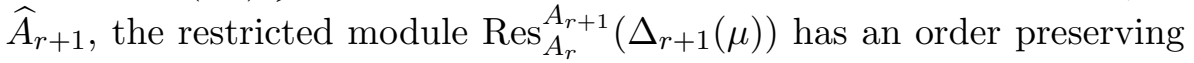
cell-filtration.

(2) A tower $\left(A_{r}\right)_{r \geqslant 0}$ is induction-coherent if for each $r \geqslant 0$ and each $\lambda \epsilon$ $\widehat{A}_{r}$, the induced module $\operatorname{Ind}_{A_{r}}^{A_{r+1}}\left(\Delta_{r}^{R}(\lambda)\right)$ has an order preserving cellfiltration.

(3) The tower $\left(A_{r}\right)_{r \geqslant 0}$ is coherent if it is both restriction- and inductioncoherent.

Remark 2.7. We have changed the terminology from [4, 6, 7], as the weaker notion of coherence, in which the order preserving requirement is omitted, plays no role here.

We now list the first of our assumptions for a sequence of diagram algebras:

(D1) $A_{0}=R$.

(D2) The algebras $A_{r}$ are cyclic cellular for all $r \geqslant 0$.

For all $k$ and for all $\lambda \in \widehat{A}_{k}$, fix once and for all a bimodule isomorphism $\alpha_{\lambda}: A_{k}^{\triangleright \lambda} / A_{k}^{\triangleright \lambda} \rightarrow\left(\Delta_{k}(\lambda)\right)^{*} \otimes_{R} \Delta_{k}(\lambda)$, a generator $\delta_{k}(\lambda)$ of the cyclic $A_{k}$-module $\Delta_{k}(\lambda)$, and an element $m_{\lambda} \in A_{k}^{\triangleright \lambda}$ satisfying $\alpha_{\lambda}\left(m_{\lambda}+A_{k}^{\triangleright \lambda}\right)=$ $\left(\delta_{k}(\lambda)\right)^{*} \otimes \delta_{k}(\lambda)$. Recall the properties of $m_{\lambda}$ from Lemma 2.4. We require the following mild assumption on the elements $m_{\lambda}$.

(D3) $m_{\lambda}=m_{\lambda}^{*}$.

Our list of assumptions continues as follows:

(D4) $A_{r}^{\mathbb{F}}$ is split semisimple for all $r \geqslant 0$.

(D5) The sequence of algebras $\left(A_{r}\right)_{r \geqslant 0}$ is restriction-coherent.

As discussed in [4, Section 3], under the assumptions (D1)-(D5) above, there exists a well defined multiplicity-free branching diagram $\widehat{A}$ associated with the sequence $\left(A_{r}\right)_{r \geqslant 0}$. The branching diagram is an infinite, graded, directed graph with vertices $\widehat{A}_{r}$ at level $k$ and edges determined as follows. For $\lambda \in \widehat{A}_{r-1}$ and $\mu \in \widehat{A}_{r}$, there is an edge $\lambda \rightarrow \mu$ in $\widehat{A}$ if and only if $\Delta_{r-1}(\lambda)$ appears as a subquotient of an order preserving cell filtration of $\operatorname{Res}_{A_{r-1}}^{A_{r}}\left(\Delta_{r}(\mu)\right)$. Note that $\widehat{A}_{0}$ is a singleton; we denote its unique element by $\varnothing$. We can choose $\Delta_{0}(\varnothing)=R, \delta_{0}(\varnothing)=1$, and $m_{\varnothing}=1$.

Definition 2.8. Given $\nu \in \widehat{A}_{r}$, we define a standard tableau of shape $\nu$ to be a directed path $\mathrm{t}$ on the branching diagram $\widehat{A}$ from $\varnothing \in \widehat{A}_{0}$ to $\nu$,

$$
\mathrm{t}=(\varnothing=\mathrm{t}(0) \rightarrow \mathrm{t}(1) \rightarrow \mathrm{t}(2) \rightarrow \cdots \rightarrow \mathrm{t}(r-1) \rightarrow \mathrm{t}(r)=\nu) .
$$


We let $\operatorname{Std}_{r}(\nu)$ denote the set of all such paths and let $\operatorname{Std}_{r}=\cup_{\nu \in \widehat{A}_{r}} \operatorname{Std}_{r}(\nu)$.

It is shown in in $[4$, Section 3] that there exist certain "branching factors" $d_{\lambda \rightarrow \mu} \in A_{r}$ associated to each edge $\lambda \rightarrow \mu$ in $\widehat{A}$, related to the cell filtration of $\operatorname{Res}_{A_{r-1}}^{A_{r}}\left(\Delta_{r}(\mu)\right)$. Given a path $\mathrm{t} \in \operatorname{Std}_{r}(\nu)$,

$$
\varnothing=\mathrm{t}(0) \rightarrow \mathrm{t}(1) \rightarrow \mathrm{t}(2) \rightarrow \cdots \rightarrow \mathrm{t}(r-1) \rightarrow \mathrm{t}(r)=\nu,
$$

define $d_{\mathrm{t}}=d_{\mathrm{t}(r-1) \rightarrow \mathrm{t}(r)} d_{\mathrm{t}(r-2) \rightarrow \mathrm{t}(r-1)} \cdots d_{\mathrm{t}(0) \rightarrow \mathrm{t}(1)}$.

We say two cellular bases of an algebra $A$ with involution are equivalent if they determine the same two sided ideals $A^{\unrhd \lambda}$ and isomorphic cell modules.

Theorem 2.9 ([4], Section 3$)$. Let $\left(A_{r}\right)_{r \geqslant 0}$ be a sequence of algebras satisfying assumptions (D1)-(D5).

(1) Let $\lambda \in \widehat{A}_{r}$. The set $\left\{m_{\lambda} d_{\mathrm{t}}+A_{r}^{\triangleright \lambda} \mid \mathrm{t} \in \operatorname{Std}_{r}(\lambda)\right\}$ is a basis of the cell module $\Delta_{r}(\lambda)$.

(2) The set $\left\{d_{\mathrm{s}}^{*} m_{\lambda} d_{\mathrm{t}} \mid \lambda \in \widehat{A}_{r}, \mathrm{~s}, \mathrm{t} \in \operatorname{Std}_{r}(\lambda)\right\}$ is a cellular basis of $A_{r}$, equivalent to the original cellular basis.

(3) For a fixed $\lambda \in \widehat{A}_{r}$, we let $\mu(1) \triangleright \mu(2) \triangleright \cdots \triangleright \mu(s)$ be a listing of the $\mu \in \widehat{A}_{r-1}$ such that $\mu \rightarrow \lambda$. Let

$$
M_{j}=\operatorname{Span}_{R}\left\{m_{\lambda} d_{\mathrm{t}}+A_{r}^{\triangleright \lambda} \mid \mathrm{t} \in \operatorname{Std}_{r}(\lambda), \mathrm{t}(k-1) \unrhd \mu(j)\right\} .
$$

Then

$$
(0) \subset M_{1} \subset \cdots \subset M_{s}=\Delta_{r}(\lambda)
$$

is a filtration of $\Delta_{r}(\lambda)$ by $A_{r-1}$ submodules, and $M_{j} / M_{j-1} \cong \Delta_{r-1}\left(\mu_{j}\right)$.

Notation 2.10. We write $m_{\mathrm{st}}^{\lambda}=d_{\mathrm{s}}^{*} m_{\lambda} d_{\mathrm{t}}$. Also write $m_{\mathrm{t}}=m_{\lambda} d_{\mathrm{t}}+A_{r}^{\triangleright \lambda} \in$ $\Delta_{r}(\lambda)$. We refer to the cellular basis $\left\{m_{\text {st }}^{\lambda} \mid \lambda \in \widehat{A}_{r}\right.$ and $\left.\mathrm{s}, \mathrm{t} \in \operatorname{Std}_{r}(\lambda)\right\}$ as the Murphy cellular basis of $A_{r}$ and $\left\{m_{\mathrm{t}}^{\lambda} \mid \mathrm{t} \in \operatorname{Std}_{r}(\lambda)\right\}$ as the Murphy basis of the cell module $\Delta_{k}(\lambda)$.

We will now continue with our list of assumed properties of the sequence of algebra $\left(A_{r}\right)_{r \geqslant 0}$ with one final axiom.

(D6) There exist ' $u$-branching factors' $u_{\mu \rightarrow \lambda} \in A_{r+1}^{R}$ such that

$$
m_{\lambda} d_{\mu \rightarrow \lambda}=\left(u_{\mu \rightarrow \lambda}\right)^{*} m_{\mu} .
$$

Example 2.11. It is shown in [4] that the Hecke algebras of type $A$, the symmetric group algebras, the Brauer algebras, the Birman-Wenzl-Murakami algebras, the partition algebras, and the Jones-Temperley-Lieb algebras all are examples of sequences of algebras satisfying properties (D1)-(D6). For the Hecke algebras, the cellular basis of Theorem 2.9 agrees with the Murphy basis from [15], up to a normalization. In each case the ground ring $R$ can be taken to be the generic ground ring for the class of algebras. For example, for the Hecke algebras, this is $\mathbb{Z}\left[\boldsymbol{q}, \boldsymbol{q}^{-1}\right]$, and for the Brauer algebras it is $\mathbb{Z}[\boldsymbol{\delta}]$, where $\boldsymbol{q}$ and $\boldsymbol{\delta}$ are indeterminants.

Let $G_{n}$ be either the general linear group $\mathrm{GL}_{n}$, the orthogonal group $\mathrm{O}_{n}$, or the symplectic group $\mathrm{Sp}_{2 n}$ and let $V$ denote its natural module. The centralizer algebra $\operatorname{End}_{G_{n}}\left(V^{\otimes r}\right)$ is a quotient of the symmetric group $\mathfrak{S}_{r}$, the Brauer algebra $B_{r}(n)$, or the Brauer algebra $B_{r}(-2 n)$ respectively. In 
each case the ground ring $R$ can be taken to be $\mathbb{Z}$. It is shown in [3] that the algebras $\operatorname{End}_{G}\left(V^{\otimes r}\right)$ all satisfy axioms (D1)-(D6).

Definition 2.12. Given $0 \leqslant s \leqslant r$ and $\lambda \in \widehat{A}_{s}, \nu \in \widehat{A}_{r}$, we define a skew standard tableau of shape $\nu \backslash \lambda$ and degree $r-s$ to be a directed path $\mathrm{t}$ on the branching diagram $\widehat{A}$ from $\lambda$ to $\nu$,

$$
\mathrm{t}=(\lambda=\mathrm{t}(s) \rightarrow \mathrm{t}(s+1) \rightarrow \mathrm{t}(s+2) \rightarrow \cdots \rightarrow \mathrm{t}(r-1) \rightarrow \mathrm{t}(r)=\nu) .
$$

We let $\operatorname{Std}_{s, r}(\nu \backslash \lambda)$ denote the set of all such paths with given $\lambda$ and $\nu$. Given $0 \leqslant s \leqslant r$, we set $\operatorname{Std}_{s, r}=\cup_{\lambda \in \widehat{A}_{s}, \nu \in \widehat{A}_{r}} \operatorname{Std}_{s, r}(\nu \backslash \lambda)$.

Given two paths $\mathbf{s} \in \operatorname{Std}_{q, s}(\mu \backslash \lambda)$ and $\mathrm{t} \in \operatorname{Std}_{s, r}(\nu \backslash \mu)$ such that the final point of $s$ is the initial point of $t$, define $s \circ t$ to be the obvious path obtained by concatenation.

Remark 2.13. Given a path $\mathrm{t} \in \operatorname{Std}_{s, r}(\nu \backslash \lambda)$ of the form

$$
\lambda=\mathrm{t}(s) \rightarrow \mathrm{t}(s+1) \rightarrow \mathrm{t}(s+2) \rightarrow \cdots \rightarrow \mathrm{t}(r-1) \rightarrow \mathrm{t}(r)=\nu,
$$

define

$$
d_{\mathrm{t}}=d_{\mathrm{t}(r-1) \rightarrow \mathrm{t}(r)} d_{\mathrm{t}(r-2) \rightarrow \mathrm{t}(r-1)} \cdots d_{\mathrm{t}(s) \rightarrow \mathrm{t}(s+1)},
$$

and

$$
u_{\mathrm{t}}=u_{\mathrm{t}(s) \rightarrow \mathrm{t}(s+1)} \cdots u_{\mathrm{t}(r-2) \rightarrow \mathrm{t}(r-1)} u_{\mathrm{t}(r-1) \rightarrow \mathrm{t}(r)} .
$$

Then it follows from the compatibility relation (3) and induction on $r-s$ that

$$
u_{\mathrm{t}}^{*} m_{\lambda}=m_{\nu} d_{\mathrm{t}} .
$$

Because $m_{\varnothing}$ can be chosen to be 1 , this gives in particular for $\mathrm{t} \in \operatorname{Std}_{r}(\nu)$,

$$
u_{\mathrm{t}}^{*}=m_{\nu} d_{\mathrm{t}} .
$$

Therefore the cellular basis $\left\{m_{\text {st }}^{\nu}\right\}$ can also be written in the apparently asymmetric form

$$
m_{\mathrm{st}}^{\nu}=d_{\mathrm{s}}^{*} m_{\nu} d_{\mathrm{t}}=d_{\mathrm{s}}^{*} u_{\mathrm{t}}^{*} .
$$

Using the symmetry of the cellular basis $\left(m_{\mathrm{st}}^{\nu}\right)^{*}=m_{\mathrm{ts}}^{\nu}$ (which follows from the assumption (D3)), we also get

$$
m_{\mathrm{st}}^{\nu}=u_{\mathrm{s}} d_{\mathrm{t}} .
$$

Using (5), we have the following form for the basis $\left\{m_{\mathrm{t}}^{\nu} \mid \mathrm{t} \in \operatorname{Std}_{r}(\nu)\right\}$ of the cell module $\Delta_{r}(\nu)$ :

$$
m_{\mathrm{t}}^{\nu}=u_{\mathrm{t}}^{*}+A_{r}^{\triangleright \nu} .
$$

Now, for any $0 \leqslant q \leqslant s \leqslant r$, let $\mathrm{t}_{[q, s]}$ denote the truncated path,

$$
\mathrm{t}(q) \rightarrow \mathrm{t}(q+1) \rightarrow \mathrm{t}(q+2) \rightarrow \cdots \rightarrow \mathrm{t}(s-1) \rightarrow \mathrm{t}(s) .
$$

The representative $u_{\mathrm{t}}^{*}$ of $m_{\mathrm{t}}$ has the remarkable property that for any $0 \leqslant$ $s \leqslant r$,

and

$$
u_{\mathrm{t}}^{*}=u_{\mathrm{t}_{[s, r]}}^{*} u_{\mathrm{t}_{[0, s]}}^{*},
$$

$$
u_{\mathrm{t}_{[0, s]}}^{*}=m_{\mathrm{t}(s)} d_{\mathrm{t}_{[0, s]}} \in m_{\mathrm{t}(s)} A_{s} \subseteq A_{s}^{\unrhd \mathrm{t}(s)} .
$$


The compatibility relations (4) together with the factorizability (7) of representatives $u_{\mathrm{t}}^{*}$ of the Murphy basis play a crucial role in this paper. They lead directly to our dominance triangularity results, which in turn lead to strong results about restriction of cell modules and allow the construction of skew cell modules. In our view, these are the distinguishing properties of the Murphy bases of diagram algebras, and even in the original context of the Hecke algebras [15] these properties provide new insight.

\section{Seminormal Basis AND DOMinance tRIANgUlarity}

3.1. Gelfand-Zeitlin idempotents. Consider an increasing sequence of algebras $\left(A_{r}\right)_{r \geqslant 0}$ satisfying assumptions (D1)-(D6) of Section 2.2. Let us recall the following notion pertaining to the tower $\left(A_{r}^{\mathbb{F}}\right)_{r \geqslant 0}$. The terminology is from Okounkov and Vershik [16, 17].

Definition 3.1. The Gelfand-Zeitlin subalgebra $G_{r}$ of $A_{r}^{\mathbb{F}}$ is the subalgebra generated by the centers of $A_{0}^{\mathbb{F}}, A_{1}^{\mathbb{F}}, \ldots, A_{r}^{\mathbb{F}}$.

The Gelfand-Zeitlin subalgebra is a maximal abelian subalgebra of $A_{r}^{\mathbb{F}}$ and contains a canonical family of idempotents indexed by paths on the branching diagram $\widehat{A}$. For each $s$ let $\left\{z_{s}^{\lambda} \mid \lambda \in \widehat{A}_{s}\right\}$ denote the set of minimal central idempotents in $A_{s}^{\mathbb{F}}$. For $r \geqslant 1$ and $\mathrm{t}$ a path on $\widehat{A}$ of length $r$, let $F_{\mathrm{t}}=\prod_{s=1}^{r} z_{s}^{\mathrm{t}(s)}$. The elements $F_{\mathrm{t}}$ for $\mathrm{t} \in \operatorname{Std}_{r}$ are mutually orthogonal minimal idempotents whose sum is the identity; moreover $\sum_{\mathrm{t} \in \operatorname{Std}_{r}(\lambda)} F_{\mathrm{t}}=$ $z_{r}^{\lambda}$. If $\mathrm{s} \in \operatorname{Std}_{s}$ and $\mathrm{t} \in \operatorname{Std}_{r}$ with $s \leqslant r$, then $F_{\mathrm{s}} F_{\mathrm{t}}=\delta_{\mathrm{s}, \mathrm{t}[0, s]} F_{\mathrm{t}}$. Evidently, the set $\left\{F_{\mathrm{t}} \mid \mathrm{t} \in \operatorname{Std}_{s}, 0 \leqslant s \leqslant r\right\}$ generates $G_{r}$. Let us call this set of idempotents the family of Gelfand-Zeitlin idempotents for $\left(A_{r}^{\mathbb{F}}\right)_{r \geqslant 0}$.

3.2. Seminormal bases. Let $\left\{m_{\mathrm{st}}^{\lambda} \mid \lambda \in \widehat{A}_{r}\right.$ and $\left.\mathrm{s}, \mathrm{t} \in \operatorname{Std}_{r}(\lambda)\right\}$ be the Murphy cellular basis of $A_{r}$ constructed in Section 2.2, and for $\lambda \in \widehat{A}_{r}$, let $\left\{m_{\mathrm{t}}^{\lambda} \mid \mathrm{t} \in \operatorname{Std}_{r}(\lambda)\right\}$ be the Murphy basis of the cell module $\Delta_{r}(\lambda)$. For $\mathrm{t} \in \operatorname{Std}_{r}$, let $F_{\mathrm{t}}$ be the corresponding Gelfand-Zeitlin idempotent in $A_{r}^{\mathbb{F}}$.

Definition 3.2 (Seminormal bases). Let $r \geqslant 0$ and $\lambda \in \widehat{A}_{r}$. For $\mathrm{s}, \mathrm{t} \in$ $\operatorname{Std}_{r}(\lambda)$, define $f_{\mathrm{t}}^{\lambda}=m_{\mathrm{t}} F_{\mathrm{t}} \in \Delta_{r}^{\mathbb{F}}(\lambda)$ and $F_{\mathrm{st}}^{\lambda}=F_{\mathrm{s}} m_{\mathrm{st}}^{\lambda} F_{\mathrm{t}} \in A_{r}^{\mathbb{F}}$.

We now define two partial orders the sets of paths $\operatorname{Std}_{s, r}$ in the branching graph.

Definition 3.3 (Dominance order). For $\mathrm{s}, \mathrm{t} \in \operatorname{Std}_{s, r}$, define $\mathrm{s} \unrhd \mathrm{t}$ if $\mathrm{s}(j) \unrhd$ $\mathrm{t}(j)$ for all $0 \leqslant j \leqslant r$. We write $\mathrm{s} \triangleright \mathrm{t}$ if $\mathrm{s} \neq \mathrm{t}$ and $\mathrm{s} \unrhd \mathrm{t}$.

This is evidently a partial order, which we call the dominance order on paths. In particular, the dominance order is defined on $\operatorname{Std}_{r}$ and on $\operatorname{Std}_{r}(\lambda)$ for $\lambda \in \widehat{A}_{r}$.

Definition 3.4 (Reverse lexicographic order). For $\mathrm{s}, \mathrm{t} \in \operatorname{Std}_{s, r}$, define $\mathrm{s} \succcurlyeq \mathrm{t}$ if $\mathrm{s}=\mathrm{t}$ or if for the last index $j$ such that $\mathrm{s}(j) \neq \mathrm{t}(j)$, we have $\mathrm{s}(j) \triangleright \mathrm{t}(j)$. We write $\mathrm{s} \succ \mathrm{t}$ if $\mathrm{s} \neq \mathrm{t}$ and $\mathrm{s} \succcurlyeq \mathrm{t}$.

This is also a partial order on paths (and is defined on $\operatorname{Std}_{r}$ and on $\operatorname{Std}_{r}(\lambda)$ for $\left.\lambda \in \widehat{A}_{r}\right)$. Evidently $\mathrm{s} \triangleright \mathrm{t}$ implies $\mathrm{s} \succ \mathrm{t}$. 
Theorem 3.5 (Dominance triangularity). Fix $\lambda \in \widehat{A}_{r}$. For all $\mathrm{t} \in \operatorname{Std}_{r}(\lambda)$, there exist coefficients $r_{\mathrm{s}}, r_{\mathrm{s}}^{\prime} \in \mathbb{F}$ such that

$$
m_{\mathrm{t}}^{\lambda}=f_{\mathrm{t}}^{\lambda}+\sum_{\substack{\mathrm{s} \in \operatorname{Std}_{r}(\lambda) \\ \mathbf{s} \triangleright \mathrm{t}}} r_{\mathrm{s}} f_{\mathrm{s}}^{\lambda} \quad f_{\mathrm{t}}^{\lambda}=m_{\mathrm{t}}^{\lambda}+\sum_{\substack{\mathbf{s} \in \operatorname{Std}_{r}(\lambda) \\ \mathbf{s} \triangleright \mathrm{t}}} r_{\mathrm{s}}^{\prime} m_{\mathrm{s}}^{\lambda} .
$$

In particular, $\left\{f_{\mathrm{t}}^{\lambda} \mid \mathrm{t} \in \operatorname{Std}_{r}(\lambda)\right\}$ is a basis of $\Delta_{r}^{\mathbb{F}}(\lambda)$.

Proof. The element $\sum_{\mu \unrhd \lambda} z_{s}^{\mu}$ acts as the identity on the ideal $A_{s}^{\unrhd \lambda}$. For $\lambda \in \widehat{A}_{r}$ and $\mathrm{t} \in \operatorname{Std}_{r}(\lambda), u_{\mathrm{t}}^{*}$ has the property that $u_{\mathrm{t}_{[0, s]}^{*}}^{*} \in A_{s}^{\unrhd \mathrm{t}(s)}$ for all $0 \leqslant s \leqslant r$, and $u_{\mathrm{t}}^{*}=u_{\mathrm{t}_{[s, r]}}^{*} u_{\mathrm{t}_{[0, s]}}^{*}$. We therefore have that

$$
u_{\mathrm{t}}^{*}=u_{\mathrm{t}}^{*} \sum_{\mu(s) \unrhd \mathrm{t}(s)} z_{s}^{\mu(s)} .
$$

Applying this at each $0 \leqslant s \leqslant r$ gives

$$
u_{\mathrm{t}}^{*}=u_{\mathrm{t}}^{*} \prod_{1 \leqslant s \leqslant r} \sum_{\mu(s) \triangleq \mathrm{t}(s)} z_{s}^{\mu(s)}=u_{\mathrm{t}}^{*} \sum_{(\mu(1), \mu(2), \ldots, \mu(r))} \prod_{1 \leqslant s \leqslant r} z_{s}^{\mu(s)}
$$

where the sum is over all sequences $(\mu(1), \mu(2), \ldots, \mu(r))$ such that $\mu(s) \in \widehat{A}_{s}$ and $\mu(s) \triangleq \mathrm{t}(s)$ for all $0 \leqslant s \leqslant r$. If such a sequence is not an element of $\operatorname{Std}_{r}$, i.e. is not a path on $\widehat{A}$, then the product $\prod_{1 \leqslant s \leqslant r} z_{s}^{\mu(s)}$ is zero. On the other hand, if

$$
\mathrm{s}=(\mu(1) \rightarrow \mu(2) \rightarrow \cdots \rightarrow \mu(r))
$$

is an element of $\operatorname{Std}_{r}$, then $\prod_{1 \leqslant s \leqslant r} z_{s}^{\mu(s)}=F_{\mathbf{s}}$. Thus

$$
u_{\mathrm{t}}^{*}=u_{\mathrm{t}}^{*} \sum_{\substack{\mathbf{s} \in \operatorname{Std}_{r} \\ \mathbf{s} \geqslant \mathrm{t}}} F_{\mathrm{s}}=u_{\mathrm{t}}^{*} F_{\mathrm{t}}+\sum_{\substack{\mathbf{s} \in \operatorname{Std}_{r}(\lambda) \\ \mathbf{s} \triangleright \mathrm{t}}} u_{\mathrm{t}}^{*} F_{\mathbf{s}}+y,
$$

where $y \in\left(A_{r}^{\mathbb{F}}\right)^{\triangleright \lambda}$. Passing to the cell module $\Delta_{r}^{\mathbb{F}}(\lambda)$ we have

$$
m_{\mathrm{t}}^{\lambda}=f_{\mathrm{t}}^{\lambda}+\sum_{\substack{\mathbf{s} \in \operatorname{Std}_{r}(\lambda) \\ \mathbf{s} \triangleright \mathrm{t}}} m_{\mathrm{t}}^{\lambda} F_{\mathrm{s}}
$$

But the range of $F_{s}$ acting on the simple module $\Delta_{r}^{\mathbb{F}}(\lambda)$ is of dimension 1 , spanned by $f_{\mathrm{s}}^{\lambda}$, so this gives

$$
m_{\mathrm{t}}^{\lambda}=f_{\mathrm{t}}^{\lambda}+\sum_{\substack{\mathbf{s} \in \operatorname{Std}_{r}(\lambda) \\ \mathbf{s} \triangleright \mathrm{t}}} r_{\mathrm{s}} f_{\mathrm{s}}^{\lambda},
$$

for appropriate $r_{\mathbf{s}} \in \mathbb{F}$. This shows that the tuple $\left[m_{\mathrm{t}}^{\lambda}\right]_{\mathrm{t} \in \operatorname{Std}_{r}(\lambda)}$ is related to the tuple $\left[f_{\mathrm{t}}^{\lambda}\right]_{\mathrm{t} \in \operatorname{Std}_{r}(\lambda)}$ by an (invertible) matrix which is unitriangular with respect to dominance order of paths. Hence $\left\{f_{\mathrm{t}}^{\lambda} \mid \mathrm{t} \in \operatorname{Std}_{r}(\lambda)\right\}$ is a basis of $\Delta_{r}^{\mathbb{F}}(\lambda)$ and the inverse change of basis matrix is also unitriangular.

Corollary 3.6. For $r \geqslant 0$, we have that

(1) $\left\{f_{\mathrm{t}}^{\lambda} \mid \mathrm{t} \in \operatorname{Std}_{r}(\lambda)\right\}$ is a basis of $\Delta_{r}^{\mathbb{F}}(\lambda)$ for all $\lambda \in \widehat{A}_{r}$.

(2) $\left\{F_{\mathrm{st}}^{\lambda} \mid \lambda \in \widehat{A}_{r}\right.$ and $\left.\mathrm{s}, \mathrm{t} \in \widehat{A}_{r}\right\}$ is a cellular basis of $A_{r}^{\mathbb{F}}$. 
Proof. The first statement was verified in the proof of Theorem 3.5. The second statement follows from [7, Lemma 2.3], since $F_{\text {st }}^{\lambda}$ is a lift of $\alpha_{\lambda}^{-1}\left(f_{\mathrm{s}}^{\lambda} \otimes_{\mathbb{F}}\right.$ $\left.f_{\mathrm{t}}^{\lambda}\right)$.

We note that the cell module $\Delta_{r}^{\mathbb{F}}(\lambda)$ imbeds in the algebra $A_{r}^{\mathbb{F}}$ as a right ideal:

Lemma 3.7. Let $r \geqslant 0$ and let $\lambda \in \widehat{A}_{r}$.

(1) $\operatorname{Span}_{\mathbb{F}}\left\{u_{\mathrm{t}}^{*} F_{\mathrm{t}} \mid \mathrm{t} \in \operatorname{Std}_{r}(\lambda)\right\}$ is a right ideal of $A_{r}^{\mathbb{F}}$ and $f_{\mathrm{t}}^{\lambda} \mapsto u_{\mathrm{t}}^{*} F_{\mathrm{t}}$ determines an isomorphism of $\Delta_{r}^{\mathbb{F}}(\lambda)$ onto this right ideal.

(2) Likewise for any fixed $\mathbf{s} \in \operatorname{Std}_{r}(\lambda)$,

$$
\Delta_{r}^{\mathbb{F}}(\lambda) \cong \operatorname{Span}_{\mathbb{F}}\left\{m_{\text {st }}^{\lambda} F_{\mathrm{t}} \mid \mathrm{t} \in \operatorname{Std}_{r}(\lambda)\right\} \cong \operatorname{Span}_{\mathbb{F}}\left\{F_{\text {st }}^{\lambda} \mid \mathrm{t} \in \operatorname{Std}_{r}(\lambda)\right\},
$$

with isomorphisms determined by $f_{\mathrm{t}}^{\lambda} \mapsto m_{\mathrm{st}}^{\lambda} F_{\mathrm{t}}$, respectively $f_{\mathrm{t}}^{\lambda} \mapsto F_{\mathrm{st}}^{\lambda}$.

Proof. Recall that $\delta_{\lambda}$ is a generator of the cell module $\Delta_{r}^{\mathbb{F}}(\lambda)$ and $m_{\lambda}$ is a lift in $\left(A_{r}^{\mathbb{F}}\right)^{\unrhd \lambda}$ of $\alpha_{\lambda}^{-1}\left(\left(\delta_{\lambda}\right)^{*} \otimes_{\mathbb{F}} \delta_{\lambda}\right)$. For any fixed $x \in A_{r}^{\mathbb{F}}$ such that $\delta_{\lambda} x \neq 0$,

$$
f_{\mathrm{t}}^{\lambda} \mapsto \alpha_{\lambda}^{-1}\left(x^{*}\left(\delta_{\lambda}\right)^{*} \otimes_{\mathbb{F}} f_{\mathrm{t}}^{\lambda}\right)=x^{*} m_{\lambda} d_{\mathrm{t}} F_{\mathrm{t}}+\left(A_{r}^{\mathbb{F}}\right)^{\triangleright \lambda}
$$

determines an isomorphism of $\Delta_{k}^{\mathbb{F}}(\lambda)$ onto a submodule of $\left(A_{r}^{\mathbb{F}}\right)^{\unrhd \lambda} /\left(A_{r}^{\mathbb{F}}\right)^{\triangleright \lambda}$. Therefore, for $b \in A_{r}^{\mathbb{F}}$, if $f_{\mathrm{t}}^{\lambda} b=\sum_{\mathrm{s}} \beta_{\mathrm{s}} f_{\mathrm{s}}^{\lambda}$, then

$$
x^{*} m_{\lambda} d_{\mathrm{t}} F_{\mathrm{t}} b=\sum_{\mathrm{s}} \beta_{\mathrm{s}} x^{*} m_{\lambda} d_{\mathrm{s}} F_{\mathrm{s}}+y,
$$

where $y \in\left(A_{r}^{\mathbb{F}}\right)^{\triangleright \lambda}$. Now for all $\mathrm{s} \in \operatorname{Std}_{r}(\lambda), F_{\mathrm{s}} z_{k}^{\lambda}=F_{\mathrm{s}}$, but $y z_{k}^{\lambda}=0$. Thus multiplying by $z_{k}^{\lambda}$ on the right gives

$$
x^{*} m_{\lambda} d_{\mathrm{t}} F_{\mathrm{t}} b=\sum_{\mathrm{s}} \beta_{\mathrm{s}} x^{*} m_{\lambda} d_{\mathrm{s}} F_{\mathrm{s}},
$$

which shows that $\operatorname{Span}_{\mathbb{F}}\left\{x^{*} m_{\lambda} d_{\mathrm{t}} F_{\mathrm{t}} \mid \mathrm{t} \in \operatorname{Std}_{r}(\lambda)\right\}$ is a right ideal and $f_{\mathrm{t}} \mapsto$ $x^{*} m_{\lambda} d_{\mathrm{t}} F_{\mathrm{t}}$ determines an isomorphism of $\Delta_{k}^{\mathbb{F}}(\lambda)$ onto this right ideal. Taking $x=1$ yields statement (1), and taking $x=d_{\mathrm{s}}$, respectively $x=d_{\mathrm{s}} F_{\mathrm{s}}$, gives the isomorphisms in part (2).

Lemma 3.8. Let $r \geqslant 0, \lambda, \mu \in \widehat{A}_{r}, \mathrm{~s}, \mathrm{t} \in \operatorname{Std}_{r}(\lambda)$, and $\mathrm{u}, \mathrm{v} \in \operatorname{Std}_{r}(\mu)$.

(1) $f_{\mathrm{t}}^{\lambda} F_{\mathrm{uv}}^{\mu}=\delta_{\lambda, \mu}\left\langle f_{\mathrm{t}}^{\lambda}, f_{\mathrm{u}}^{\lambda}\right\rangle f_{\mathrm{v}}^{\lambda}$.

(2) $F_{\mathrm{st}}^{\lambda} F_{\mathrm{uv}}^{\mu}=\delta_{\lambda, \mu}\left\langle f_{\mathrm{t}}^{\lambda}, f_{\mathrm{u}}^{\lambda}\right\rangle F_{\mathrm{sv}}^{\lambda}$.

(3) $\left\langle f_{\mathrm{t}}^{\lambda}, f_{\mathrm{u}}^{\lambda}\right\rangle \neq 0$ if and only if $\mathrm{t}=\mathrm{u}$.

(4) $\left\langle f_{\mathrm{t}}^{\lambda}, f_{\mathrm{t}}^{\lambda}\right\rangle^{-1} F_{\mathrm{tt}}^{\lambda}=F_{\mathrm{t}}$.

(5) The set of elements $E_{\mathrm{st}}^{\lambda}=\left\langle f_{\mathrm{s}}^{\lambda}, f_{\mathrm{s}}^{\lambda}\right\rangle^{-1} F_{\mathrm{st}}^{\lambda}$ for $\lambda \in \widehat{A}_{r}$ and $\mathrm{s}, \mathrm{t} \in \operatorname{Std}_{r}(\lambda)$ is a complete family of matrix units with

$$
E_{\mathrm{st}}^{\lambda} E_{\mathrm{uv}}^{\mu}=\delta_{\lambda, \mu} \delta_{\mathrm{t}, \mathrm{u}} E_{\mathrm{sv}}^{\lambda}, \quad \text { and } \quad E_{\mathrm{st}}^{\lambda} E_{\mathrm{ts}}^{\lambda}=E_{\mathrm{ss}}^{\lambda}=F_{\mathrm{s}} .
$$

Proof. If $\lambda \neq \mu$, then $f_{\mathrm{t}}^{\lambda} F_{\mathrm{uv}}^{\mu}=f_{\mathrm{t}}^{\lambda} F_{\mathrm{t}} F_{\mathrm{u}} F_{\mathrm{uv}}^{\mu}=0$, and similarly $F_{\mathrm{st}}^{\lambda} F_{\mathrm{uv}}^{\mu}=0$. We have $f_{\mathrm{t}}^{\lambda} F_{\mathrm{uv}}^{\lambda}=\left\langle f_{\mathrm{t}}^{\lambda}, f_{\mathrm{u}}^{\lambda}\right\rangle f_{\mathrm{v}}^{\lambda}$ by the definition of the bilinear form (2), since $F_{\mathrm{uv}}^{\lambda}$ is a lift of $\alpha_{\lambda}^{-1}\left(\left(f_{\mathrm{u}}^{\lambda}\right)^{*} \otimes f_{\mathrm{v}}^{\lambda}\right)$. This proves part (1) and part (2) follows from Lemma 3.7 part (2). If $\mathrm{t} \neq \mathrm{u}$, then

$$
\left\langle f_{\mathrm{t}}^{\lambda}, f_{\mathrm{u}}^{\lambda}\right\rangle=\left\langle f_{\mathrm{t}}^{\lambda} F_{\mathrm{t}}, f_{\mathrm{u}}^{\lambda} F_{\mathrm{u}}\right\rangle=\left\langle f_{\mathrm{t}}^{\lambda} F_{\mathrm{t}} F_{\mathrm{u}}, f_{\mathrm{u}}^{\lambda}\right\rangle=0 .
$$


Suppose that $\left\langle f_{\mathrm{t}}^{\lambda}, f_{\mathrm{t}}^{\lambda}\right\rangle=0$ for some $\mathrm{t} \in \operatorname{Std}_{r}(\lambda)$. Then it follows from part (2) and the orthogonality of the elements $f_{u}^{\lambda}$ which was just established, that $f_{\mathrm{t}}^{\lambda} F_{\mathrm{uv}}^{\mu}=0$ for all $\mu$ and all $\mathrm{u}, \mathrm{v} \in \operatorname{Std}_{r}(\mu)$. But by Corollary 3.6, the identity element of $A_{r}^{\mathbb{F}}$ is in the span of the set of $F_{\mathrm{uv}}^{\mu}$, so it follows that $f_{\mathrm{t}}^{\lambda}=0$, a contradiction. This proves part (3). By part (2), $G_{\mathrm{t}}=\left\langle f_{\mathrm{t}}^{\lambda}, f_{\mathrm{t}}^{\lambda}\right\rangle^{-1} F_{\mathrm{tt}}^{\lambda}$ is an idempotent such that $G_{\mathrm{t}} F_{\mathrm{t}}=G_{\mathrm{t}}$. Since $F_{\mathrm{t}}$ is a minimal idempotent, it follows that $G_{\mathrm{t}}=F_{\mathrm{t}}$, which proves part (4). Part (5) follows from parts (2), (3), and (4).

3.3. Restriction of the seminormal representations. For $0 \leqslant s<r$ write $A_{r}^{\mathbb{F}} \cap\left(A_{s}^{\mathbb{F}}\right)^{\prime}$ for the set of $x \in A_{r}^{\mathbb{F}}$ that commute pointwise with $A_{s}^{\mathbb{F}}$.

Proposition 3.9. Let $1 \leqslant s<r$. Let $\nu \in \widehat{A}_{r}$ and $\mathrm{t} \in \operatorname{Std}_{r}(\nu)$. Write $\lambda=\mathrm{t}(s), \mathrm{t}_{1}=\mathrm{t}_{[0, s]} \in \operatorname{Std}_{s}(\lambda)$, and $\mathrm{t}_{2}=\mathrm{t}_{[s, r]} \in \operatorname{Std}_{s, r}(\nu \backslash \lambda)$.

(1) Let $x \in A_{s}^{\mathbb{F}}$ and suppose $f_{\mathbf{t}_{1}}^{\lambda} x=\sum_{\mathbf{s} \in \operatorname{Std}_{s}(\lambda)} \alpha_{\mathbf{s}} f_{\mathbf{s}}^{\lambda}$. We have that

$$
f_{\mathrm{t}}^{\nu} x=\sum_{\mathbf{s} \in \operatorname{Std}_{s}(\lambda)} \alpha_{\mathrm{s}} f_{\mathbf{s o t}_{2}}^{\nu} .
$$

(2) In particular, for $\mu \in \widehat{A}_{s}$ and $\mathrm{u}, \mathrm{v} \in \operatorname{Std}_{s}(\mu)$,

$$
f_{\mathrm{t}}^{\nu} F_{\mathrm{uv}}^{\mu}=\delta_{\mu, \lambda} \delta_{\mathrm{t}_{1}, \mathrm{u}}\left\langle f_{\mathrm{t}_{1}}^{\lambda}, f_{\mathrm{t}_{1}}^{\lambda}\right\rangle f_{\mathrm{vot}_{2}}^{\nu} .
$$

(3) For $x \in A_{r}^{\mathbb{F}} \cap\left(A_{s}^{\mathbb{F}}\right)^{\prime}, f_{\mathrm{t}}^{\nu} x=\sum_{\mathrm{s}} r_{\mathrm{s}} f_{\mathrm{t}_{1} \circ s}^{\nu}$, where the sum is over $\mathrm{s} \in \operatorname{Std}_{s, r}(\nu \backslash$ $\lambda)$, and the coefficients depend only on $x$ and $\mathrm{t}_{2}$, and are independent of $\mathrm{t}_{1}$.

Proof. We can embed the cell module $\Delta_{r}^{\mathbb{F}}(\nu)$ in $A_{r}^{\mathbb{F}}$, identifying $f_{\mathrm{t}}^{\nu}$ with $u_{\mathrm{t}}^{*} F_{\mathrm{t}}$, using Lemma 3.7. We can write $F_{\mathrm{t}}=F_{\mathrm{t}_{1}} F_{\mathrm{t}_{2}}$, where $F_{\mathrm{t}_{2}} \in A_{r}^{\mathbb{F}} \cap\left(A_{s}^{\mathbb{F}}\right)^{\prime}$. Thus

$$
f_{\mathrm{t}}^{\nu} x=u_{\mathrm{t}}^{*} F_{\mathrm{t}} x=u_{\mathrm{t}_{2}}^{*} F_{\mathrm{t}_{2}} u_{\mathrm{t}_{1}}^{*} F_{\mathrm{t}_{1}} x .
$$

Applying Lemma 3.7 part (1) to $\Delta_{s}^{\mathbb{F}}(\lambda)$ we find that this equals

$$
u_{\mathrm{t}_{2}}^{*} F_{\mathrm{t}_{2}} \sum_{\mathrm{s}} \alpha_{\mathrm{s}} u_{\mathrm{s}}^{*} F_{\mathrm{s}}=\sum_{\mathrm{s}} \alpha_{\mathrm{s}} u_{\mathrm{t}_{2}}^{*} u_{\mathrm{s}}^{*} F_{\mathrm{s}} F_{\mathrm{t}_{2}}=\sum_{\mathrm{s}} \alpha_{\mathrm{s}} u_{\mathrm{sot}_{2}}^{*} F_{\mathrm{sot}_{2}}=\sum_{\mathrm{s}} \alpha_{\mathrm{s}} f_{\mathrm{sot}_{2}}^{\nu} .
$$

This proves part (1) and part (2) is an immediate consequence. We now consider part (3). Given $x \in A_{r}^{\mathbb{F}} \cap\left(A_{s}^{\mathbb{F}}\right)^{\prime}$, we let $f_{\mathrm{t}}^{\nu} x=\sum_{\mathrm{u} \in \operatorname{Std}_{r}(\nu)} r_{\mathrm{u}} f_{\mathrm{u}}^{\nu}$. Since $x$ commutes with $A_{s}^{\mathbb{F}}$,

$$
f_{\mathrm{t}}^{\nu} x=f_{\mathrm{t}}^{\nu} F_{\mathrm{t}_{1}} x=f_{\mathrm{t}}^{\nu} x F_{\mathrm{t}_{1}}=\sum_{\mathrm{u}} r_{\mathrm{u}} f_{\mathrm{u}}^{\nu} F_{\mathrm{t}_{1}},
$$

which shows that $r_{\mathrm{u}}=0$ unless $\mathrm{u}_{[0, s]}=\mathrm{t}_{1}$. Thus, we can rewrite this as $f_{\mathrm{t}} x=\sum_{\mathrm{s}} r_{\mathrm{s}} f_{\mathrm{t}_{1} \circ s}$, where now the sum is over $\mathrm{s} \in \operatorname{Std}_{s, r}(\nu \backslash \lambda)$. It remains to show that the coefficients are independent of $\mathrm{t}_{1}$. If $v \in \operatorname{Std}_{s}(\lambda)$, then

$$
f_{\mathrm{vot}_{2}}^{\nu} x=f_{\mathrm{t}_{1} \circ \mathrm{t}_{2}}^{\nu} E_{\mathrm{t}_{1} \mathrm{v}}^{\lambda} x=f_{\mathrm{t}}^{\nu} E_{\mathrm{t}_{1} \mathrm{v}}^{\lambda} x=f_{\mathrm{t}}^{\nu} x E_{\mathrm{t}_{1} \mathrm{v}}^{\lambda}=\sum_{\mathrm{s}} r_{\mathrm{s}} f_{\mathrm{t}_{1} \circ \mathrm{s}}^{\nu} E_{\mathrm{t}_{1} \mathrm{v}}^{\lambda}=\sum_{\mathrm{s}} r_{\mathrm{s}} f_{\mathrm{vos}^{\prime}}^{\nu},
$$

where we have applied part (2). 


\subsection{Dominance triangularity and restriction rules for the Murphy} basis. For $0 \leqslant s<r$, we now show that the Murphy basis is compatible with restriction to the subalgebra $A_{s} \subseteq A_{r}$, and also to the subalgebra $A_{r} \cap A_{s}^{\prime}$. This is a first step towards constructing skew cell modules in the next section.

Lemma 3.10. Let $1 \leqslant s<r, \nu \in \widehat{A}_{r}, \lambda \in \widehat{A}_{s}$ and $\mathrm{t} \in \operatorname{Std}_{s, r}(\nu \backslash \lambda)$. Suppose that $y \in m_{\lambda} A_{s} \cap A_{s}^{\triangleright \lambda}$. Then there exist coefficients $r_{\mathrm{z}} \in R$ such that

$$
u_{\mathrm{t}}^{*} y \equiv \sum_{\substack{\mathrm{z} \in \operatorname{Std}_{r}(\nu) \\ \mathrm{z}_{[s, r]} \triangleright \mathrm{t} \\ \mathrm{z}(s) \triangleright \lambda}} r_{\mathrm{z}} u_{\mathrm{z}}^{*} \quad \bmod A_{r}^{\triangleright \nu} .
$$

Proof. Recall that the element $\sum_{\lambda(s) \triangleright \lambda} z_{s}^{\lambda(s)}$ acts as the identity on the ideal $A_{s}^{\triangleright \lambda}$ and that $y \in A_{s}^{\triangleright \lambda}$. By assumption $y \in m_{\lambda} A_{s}$ and $\mathrm{t}(s)=\lambda$, we have

$$
u_{\mathrm{t}_{[s, j]}}^{*} y \in m_{\mathrm{t}(j)} A_{j} \subseteq A_{j}^{\unrhd \mathrm{t}(j)}
$$

for each $j$ with $s<j \leqslant r$. In fact, if $y=m_{\lambda} x$, then $u_{\mathrm{t}_{[s, j]}}^{*} y=u_{\mathrm{t}_{[s, j]}}^{*} m_{\lambda} x=$ $m_{\mathrm{t}(j)} d_{\mathrm{t}_{[s, j]}} x$, using (4). It follows that $u_{\mathrm{t}}^{*} y=u_{\mathrm{t}}^{*} y\left(\sum_{\lambda(j) \unrhd \mathrm{t}(j)} z_{j}^{\lambda(j)}\right)$. Finally, for $j<s$, we can write $1=\sum_{\lambda(j) \in \widehat{A}_{j}} z_{j}^{\lambda(j)}$. Arguing as in the proof of Theorem 3.5, we have that

$$
u_{\mathrm{t}}^{*} y=\sum_{\substack{\mathrm{z} \in \operatorname{Std}_{r} \\ \mathrm{z}_{[s, r]} \triangleright \mathrm{t} \\ \mathrm{z}(s) \triangleright \lambda}} u_{\mathrm{t}}^{*} y F_{\mathrm{z}} \equiv \sum_{\substack{\mathrm{z} \in \operatorname{Std}_{r}(\nu) \\ \mathbf{z}_{[s, r]} \triangleright \mathrm{t} \\ \mathrm{z}(s) \triangleright \lambda}} u_{\mathrm{t}}^{*} y F_{\mathrm{z}} \quad \bmod \left(A_{r}^{\mathbb{F}}\right)^{\triangleright \nu} .
$$

Now, because the range of $F_{\mathbf{z}}$ on the cell module $\Delta_{r}^{\mathbb{F}}(\nu)$ is $\mathbb{F}\left\{f_{\mathrm{z}}\right\}=\mathbb{F}\left\{u_{\mathrm{z}}^{*} F_{\mathrm{z}}\right\}$, we have $u_{\mathrm{t}}^{*} y F_{\mathrm{z}}=\alpha_{\mathrm{z}} u_{\mathrm{z}}^{*} F_{\mathrm{z}}$ for some $\alpha_{\mathrm{z}} \in \mathbb{F}$. Thus

$$
u_{\mathrm{t}}^{*} y \equiv \sum_{\substack{\mathrm{z} \in \operatorname{Std}_{r}(\nu) \\ \mathbf{z}[s, r] \triangleright \mathrm{t} \\ \mathrm{z}(s) \triangleright \lambda}} \alpha_{\mathbf{z}} u_{\mathbf{z}}^{*} F_{\mathbf{z}} \quad \bmod \left(A_{r}^{\mathbb{F}}\right)^{\triangleright \nu}
$$

By dominance triangularity (Theorem 3.5) we obtain

$$
u_{\mathrm{t}}^{*} y=\sum_{\substack{\mathrm{z} \in \operatorname{Std}_{r}(\nu) \\ \mathrm{z}_{[s, r] \triangleright \mathrm{t},}, \mathrm{z}(s) \triangleright \lambda}} r_{\mathrm{z}} u_{\mathrm{z}}^{*}+y^{\prime},
$$

with coefficients $r_{\mathrm{z}} \in \mathbb{F}$, and with $y^{\prime} \in\left(A_{r}^{\mathbb{F}}\right)^{\triangleright \nu}$. But since $u_{\mathrm{t}}^{*} y \in A_{r}^{R}$, we have that $r_{\mathrm{z}} \in R$ and $y^{\prime} \in A_{r}^{\triangleright \nu}$.

The following is an immediate consequence of the lemma:

Proposition 3.11. Let $1 \leqslant s<r, \nu \in \widehat{A}_{r}, \lambda \in \widehat{A}_{s}$ and $\mathrm{t} \in \operatorname{Std}_{s, r}(\nu \backslash \lambda)$. Let $x \in m_{\lambda} A_{s}$ and write

$$
x=\sum_{\mathbf{s} \in \operatorname{Std}_{s}(\lambda)} \alpha_{\mathbf{s}} u_{\mathrm{s}}^{*}+y,
$$


with $y \in A_{s}^{\triangleright \lambda}$. Then there exist coefficients $r_{\mathrm{z}} \in R$, such that

$$
u_{\mathrm{t}}^{*} x \equiv \sum_{\mathrm{s} \in \operatorname{Std}_{s}(\lambda)} \alpha_{\mathrm{s}} u_{\mathrm{t}}^{*} u_{\mathrm{s}}^{*}+\sum_{\substack{\mathrm{z} \in \operatorname{Std}_{r}(\nu) \\ \mathrm{z}_{[s, r]} \triangleright \mathrm{t}, \mathrm{z}(s) \triangleright \lambda}} r_{\mathrm{z}} u_{\mathrm{z}}^{*} \quad \bmod A_{r}^{\triangleright \nu} .
$$

The following is a restriction rule for the Murphy type basis of a tower of diagram algebras.

Corollary 3.12. Let $r>1, \nu \in \widehat{A}_{r}$, and $\mathrm{t} \in \operatorname{Std}_{r}(\nu)$. Let $1 \leqslant s<r$ and write $\lambda=\mathrm{t}(s)$. Let $a \in A_{s}$ and suppose

$$
u_{\mathrm{t}_{[0, s]}}^{*} a \equiv \sum_{\mathrm{s} \in \operatorname{Std}_{s}(\lambda)} r_{\mathrm{s}} u_{\mathrm{s}}^{*} \bmod A_{s}^{\triangleright \lambda} .
$$

Then there exist coefficients $r_{\mathrm{z}} \in R$ such that

$$
u_{\mathrm{t}}^{*} a \equiv \sum_{\mathbf{s} \in \operatorname{Std}_{s}(\lambda)} r_{\mathrm{s}} u_{\mathrm{t}_{[s, r]}}^{*} u_{\mathrm{s}}^{*}+\sum_{\substack{\mathrm{z} \in \operatorname{Std}_{r}(\nu) \\ \mathbf{z}_{[s, r]} \triangleright \mathrm{t}, \mathrm{z}(s) \triangleright \lambda}} r_{\mathrm{z}} u_{\mathrm{z}}^{*} \quad \bmod A_{r}^{\triangleright \nu} .
$$

Proof. Apply the previous Proposition with $x=u_{\mathrm{t}_{[0, s]}}^{*} a$.

Remark 3.13. Corollary 3.12 is an improvement of the restriction rule for "path bases" obtained in [7, Proposition 2.18].

We now consider restriction to $A_{r} \cap A_{s}^{\prime} \subseteq A_{r}$.

Proposition 3.14. Let $0 \leqslant s<r, \nu \in \widehat{A}_{r}$, and $\mathrm{t} \in \operatorname{Std}_{r}(\nu)$. Write $\lambda=\mathrm{t}(s)$. Let $x \in A_{r} \cap A_{s}^{\prime}$. Then there exist coefficients $r_{\mathrm{w}} \in R$ such that

$$
u_{\mathrm{t}}^{*} x \equiv \sum_{\substack{\mathrm{w} \in \operatorname{Std}_{r}(\nu) \\ \mathrm{w}_{[0, s]} \oslash \mathrm{t}_{[0, s]}}} r_{\mathrm{w}} u_{\mathrm{w}}^{*} \quad \bmod A_{r}^{\triangleright \nu},
$$

Proof. Using Theorem 3.5, write $m_{\mathrm{t}}^{\nu}=f_{\mathrm{t}}^{\nu}+\sum_{\mathrm{u} \triangleright \mathrm{t}} \alpha_{\mathrm{u}} f_{\mathrm{u}}^{\nu}$, with coefficients $\alpha_{\mathrm{u}} \in \mathbb{F}$, so that $m_{\mathrm{t}}^{\nu} x=f_{\mathrm{t}}^{\nu} x+\sum_{\mathrm{u} \triangleright \mathrm{t}} \alpha_{\mathrm{u}} f_{\mathrm{u}}^{\nu} x$. Now applying Proposition 3.9 part (3), we see that $m_{\mathrm{t}}^{\nu} x$ is an $\mathbb{F}$-linear combination of seminormal basis elements $f_{\mathrm{v}}^{\nu}$ with $\mathrm{v}_{[0, s]} \unrhd \mathrm{t}_{[0, s]}$. Using Theorem 3.5 again, each such $f_{\mathrm{v}}^{\nu}$ is an $\mathbb{F}$-linear combination of Murphy basis elements $m_{\mathrm{w}}^{\nu}$, with $\mathrm{w} \unrhd \mathrm{v}$, and thus $\mathrm{w}_{[0, s]} \triangleq \mathrm{v}_{[0, s]} \triangleq \mathrm{t}_{[0, s]}$. Thus we get

$$
u_{\mathrm{t}}^{*} x=\sum_{\substack{\mathrm{w} \in \operatorname{Std}_{r}(\nu) \\ \mathrm{w}_{[0, s]} \triangleq \mathrm{t}_{[0, s]}}} r_{\mathrm{w}} u_{\mathrm{w}}^{*}+y,
$$

with coefficients $r_{\mathrm{w}} \in \mathbb{F}$ and with $y \in A_{r}^{\triangleright \nu} \otimes_{R} \mathbb{F}$. Since $u_{\mathrm{t}}^{*} x \in A_{r}^{R}$, the coefficients are necessarily in $R$ and $y \in A_{r}^{\triangleright \nu}$.

\section{JuCys-Murphy ELEMEnts}

We recall the definition and first properties of families of Jucys-Murphy elements for diagram algebras. The action of Jucys-Murphy elements on cell modules for diagram algebras was first considered systematically in Goodman-Graber [7], motivated by work of Mathas [12]. In this section, 
we use Theorem 3.5 to strengthen the results of [7] by replacing the reverse lexicographic order on tableaux with the dominance order on tableaux.

The treatment of Jucys-Murphy and the seminormal basis in [7] proceeds as follows. From the definition of Jucys-Murphy elements (Definitions 4.1 and 4.3) one concludes that the Jucys-Murphy elements act triangularly with respect to reverse lexicographic order on the Murphy basis. Therefore, Jucys-Murphy elements in the sense of [7] are also Jucys-Murphy elements in the sense of Mathas [12, Definition 2.4]. When the ground ring is a field $\mathbb{F}$ and Mathas' separation condition [12, Definition 2.8] is satisfied - which is true for standard examples of diagram algebras over the field of fractions of the generic ground ring - then by [12, Corollary 2.9], the algebras $A_{r}^{\mathbb{F}}$ are split semisimple. Moreover, following [12, Section 3] one can define a family orthogonal idempotents $F_{\mathrm{t}}^{\prime}$ labelled by $\mathrm{t} \in \mathrm{Std}_{r}$, using interpolation formulas for the Jucys-Murphy elements. By [7, Proposition 3.11], the idempotents $F_{\mathrm{t}}^{\prime}$ coincide with the Gelfand-Zeitlin idempotents $F_{\mathrm{t}}$ for the tower $\left(A_{r}^{\mathbb{F}}\right)_{r \geqslant 0}$. Finally, one defines the seminormal bases as in Definition 3.2. Since the $F_{\mathrm{t}}$ are polynomials in the Jucys-Murphy elements, it follows that transition matrix between the Murphy basis and the seminormal basis is unitriangular with respect to reverse lexicographic order.

Here we reverse the logic. We consider a tower of diagram algebras, i.e. a tower $\left(A_{r}\right)_{r \geqslant 0}$ satisfying (D1)-(D6). Then we already have the GelfandZeitlin idempotents at our disposal and can define the seminormal bases as in Definition 3.2. Moreover, the transition matrix between the Murphy basis and the seminormal basis is dominance unitriangular by Theorem 3.5. In case Jucys-Murphy elements in the sense of Definitions 4.1 and 4.3 exist, we will show that they act diagonally on the seminormal basis. It then follows from Theorem 3.5 that the Jucys-Murphy elements act triangularly on the Murphy basis with respect to dominance order. Assuming Mathas' separation condition holds - as it does in standard examples - one can then take up the theory in [12, Section 3], defining orthogonal idempotents by interpolation formulas using the Jucys-Murphy elements, and these must coincide with the Gelfand-Zeitlin idempotents by [7, Proposition 3.11].

For the rest of this section, assume $\left(A_{r}\right)_{r \geqslant 0}$ is a tower of diagram algebras over an integral domain $R$ with field of fractions $\mathbb{F}$, satisfying (D1)-(D6).

Definition 4.1. We say that a family of elements $\left\{L_{r} \mid r \geqslant 1\right\}$, is an additive family of Jucys-Murphy elements if the following conditions hold.

(1) For all $r \geqslant 1, L_{r} \in A_{r}, L_{r}$ is invariant under the involution of $A_{r}$, and $L_{r}$ commutes with $A_{r-1}$. In particular, $L_{i} L_{j}=L_{j} L_{i}$ for all $1 \leqslant i \leqslant j \leqslant r$.

(2) For all $r \geqslant 1$ and $\lambda \in \widehat{A}_{r}$, there exists $d(\lambda) \in R$ such that $L_{1}+\cdots+L_{r}$ acts as the scalar $d(\lambda)$ on the cell module $\Delta_{r}^{R}(\lambda)$. For $\lambda \in \widehat{A}_{0}$, we let $d(\lambda)=0$.

Example 4.2. The group algebras of symmetric groups, Temperley-Lieb, Brauer, walled Brauer, and partition algebras all possess additive families of Jucys-Murphy elements. 
Definition 4.3. We say that a family of elements $\left\{L_{r} \mid r \geqslant 1\right\}$, is a multiplicative family of Jucys-Murphy elements if the following conditions hold.

(1) For all $r \geqslant 1, L_{r}$ is an invertible element of $A_{r}, L_{r}$ is invariant under the involution $*$, and $L_{r}$ commutes with $A_{r-1}$. In particular, $L_{i} L_{j}=L_{j} L_{i}$ for all $1 \leqslant i \leqslant j \leqslant r$.

(2) For all $r \geqslant 1$ and $\lambda \in \widehat{A}_{r}$, there exists $d(\lambda) \in R$ such that $L_{1} \cdots L_{r}$ acts as the scalar $d(\lambda)$ on the cell module $\Delta_{r}^{R}(\lambda)$. For $\lambda \in \widehat{A}_{0}$, we let $d(\lambda)=1$.

Example 4.4. The Hecke algebras of finite type $A$ and the Birman-MurakamiWenzl algebra possess a multiplicative family of Jucys-Murphy elements.

Proposition 4.5. Assume that the tower $\left(A_{r}\right)_{r \geqslant 0}$ has additive or multiplicative Jucys-Murphy elements $L_{i}$. Then the Jucys-Murphy elements act diagonally on the seminormal bases. More precisely, there exist scalars $\kappa_{\mu \rightarrow \lambda} \in R$ associated to edges $\mu \rightarrow \lambda$ in $\widehat{A}$ such that for all $r, \lambda \in \widehat{A}_{r}$, $\mathrm{t} \in \operatorname{Std}_{r}(\lambda)$ and $i \leqslant r$,

$$
f_{\mathrm{t}}^{\lambda} L_{i}=\kappa_{\mathrm{t}(i-1) \rightarrow \mathrm{t}(i)} f_{\mathrm{t}}^{\lambda} .
$$

Proof. We consider the case of additive Jucys-Murphy elements. The proof for multiplicative Jucys-Murphy elements is nearly identical. Let $\kappa_{\mu \rightarrow \lambda}=$ $d(\lambda)-d(\mu)$, where $d(\cdot)$ is as in Definition 4.1. For $i \geqslant 1$ and $\mu \in \widehat{A}_{i}$, the sum $L_{1}+\cdots+L_{i}$ acts as the scalar $d(\mu)$ on $\Delta_{i}(\mu)$. It follows from the restriction rule for the seminormal representations (Proposition 3.9) that for all $r \geqslant i, \lambda \in \widehat{A}_{r}$ and $\mathrm{t} \in \operatorname{Std}_{r}(\lambda), f_{\mathrm{t}}^{\lambda}\left(L_{1}+\cdots+L_{i}\right)=d(\mathrm{t}(i)) f_{\mathrm{t}}^{\lambda}$. Hence $f_{\mathrm{t}}^{\lambda} L_{i}=\left(d(\mathrm{t}(i)-d(\mathrm{t}(i-1))) f_{\mathrm{t}}^{\lambda}\right.$.

Notation 4.6. We will also write $\kappa_{\mathrm{t}}(i)$ for $\kappa_{\mathrm{t}(i-1) \rightarrow \mathrm{t}(i)}$.

Theorem 4.7. Assume that the tower $\left(A_{r}\right)_{r \geqslant 0}$ has additive or multiplicative Jucys-Murphy elements $L_{i}$. Then the Jucys-Murphy elements act triangularly with respect to dominance order on the Murphy basis of the cell modules, i.e. for $r \geqslant 1, \lambda \in \widehat{A}_{r}$ and $\mathrm{t} \in \operatorname{Std}_{r}(\lambda)$,

$$
m_{\mathrm{t}}^{\lambda} L_{i}=\kappa_{\mathrm{t}}(i) m_{\mathrm{t}}^{\lambda}+\sum_{\mathrm{s} \triangleright \mathrm{t}} r_{\mathrm{s}} m_{\mathrm{s}}^{\lambda}
$$

where the coefficients are in $R$.

Proof. Using Proposition 4.5 and Theorem 3.5, one obtains (9) with coefficients in $\mathbb{F}$. But since $L_{i} \in A_{r}^{R}$, the coefficients are necessarily in $R$.

The dominance triangularity results Theorems 3.5 and 4.7 apply to the following examples:

(1) The symmetric group algebras and the Hecke algebras of finite type $A$. This example is discussed at length in Section 5 below.

For the remaining examples, dominance triangularity of the Jucys-Murphy elements, and of the transition matrix from a path basis or a Murphy type basis to the seminormal basis, are new results.

(2) The towers of Tempreley-Lieb algebras, partition algebras, Brauer algebras, and Birman-Murakami-Wenzl algebras [4]. 
(3) The towers of centralizer algebras on tensor space discussed in Example 2.11, [3].

\section{Applichtion to the HeCke algebras of the symmetric groups}

The arguments of Sections 3 and 4, applied to the Hecke algebras of the symmetric groups, result in some modest simplifications of the theory of these algebras, as presented, for example, in [11].

Let $S$ be an integral domain and $q \in S$ a unit. The Hecke algebra $H_{r}(S ; q)$ is the unital $S$-algebra with generators $T_{1}, \ldots, T_{r-1}$ satisfying the braid relations and the quadratic relation $\left(T_{i}-q\right)\left(T_{i}+1\right)=0$. For any $S$, the specialization $H_{r}(S ; 1)$ is isomorphic to $S \mathfrak{S}_{r}$. The generic ground ring for the Hecke algebras is the Laurent polynomial ring $R=\mathbb{Z}\left[\boldsymbol{q}, \boldsymbol{q}^{-1}\right]$, where $\boldsymbol{q}$ is an indeterminant. Let $\mathbb{F}$ denote $\mathbb{Q}(\boldsymbol{q})$, the field of fractions of $R$.

We will write $H_{r}(\boldsymbol{q})$ for $H_{r}(R ; \boldsymbol{q})$. The algebra $H_{r}(\boldsymbol{q})$ has an $R$-basis $\left\{T_{w} \mid w \in \mathfrak{S}_{r}\right\}$, defined as follows: if $w=s_{i_{1}} s_{i_{2}} \cdots s_{i_{l}}$ is a reduced expression for $w$ in the usual generators of $\mathfrak{S}_{r}$, then $T_{w}=T_{i_{1}} T_{i_{2}} \cdots T_{i_{l}}$, independent of the reduced expression. Define $T_{w}^{*}=T_{w^{-1}}$; then $*$ is an algebra involution on $H_{r}(\boldsymbol{q})$.

Let $\widehat{\mathfrak{S}}_{r}$ denote the set of Young diagrams or partitions of size $r$. Dominance order $\triangleq$ on $\widehat{\mathfrak{S}}_{r}$ is determined by $\lambda \geqslant \mu$ if $\sum_{i=1}^{j} \lambda_{i} \geqslant \sum_{i=1}^{j} \mu_{i}$ for all $1 \leqslant j \leqslant r$. Young's graph or lattice, $\widehat{\mathfrak{S}}$, is the branching diagram with vertices $\widehat{\mathfrak{S}}_{r}$ on level $r$ and a directed edge $\lambda \rightarrow \mu$ if $\mu$ is obtained from $\lambda$ by adding one box. We can identify standard tableaux of shape $\lambda$ with directed paths on $\widehat{\mathfrak{S}}$ from $\varnothing$ to $\lambda$. For $\lambda \in \widehat{\mathfrak{S}}_{r}$, denote the set of standard tableaux of shape $\lambda$ by $\operatorname{Std}_{r}(\lambda)$.

Let $\lambda$ be a Young diagram and let $\alpha=(i, j)$ be a box in $\lambda$. The content of $\alpha$ is $c(\alpha)=j-i$. If $\mathrm{t} \in \operatorname{Std}_{r}(\nu)$, and $k \leqslant r$, define $c_{\mathrm{t}}(k)=c(\alpha)$, where $\alpha=\mathrm{t}(k) \backslash \mathrm{t}(k-1)$. When $\mathrm{t}$ is regarded as an array with the boxes of the Young diagram $\nu$ filled with the numbers from 1 to $r, c_{\mathrm{t}}(k)$ is the content of the box containing the entry $k$.

For $\lambda$ a partition of $r$, let $x_{\lambda}=\sum_{w \in \mathfrak{S}_{\lambda}} T_{w}$. Let $\mathrm{t}^{\lambda}$ be the row reading tableaux of shape $\lambda$. For any $\lambda$-tableau $\mathrm{t}$, there is a unique $w(\mathrm{t}) \in \mathfrak{S}_{r}$ with $\mathrm{t}=\mathrm{t}^{\lambda} w(\mathrm{t})$. For $\lambda$ a partition of $r$ and $\mathrm{s}, \mathrm{t} \in \operatorname{Std}_{r}(\lambda)$, let

$$
x_{\mathrm{st}}^{\lambda}=\left(T_{w(\mathbf{s})}\right)^{*} x_{\lambda} T_{w(\mathrm{t})} .
$$

Theorem 5.1 (The Murphy basis, [15]). The set $\mathcal{X}=\left\{x_{\mathrm{st}}^{\lambda} \mid \lambda \in \widehat{\mathfrak{S}}_{r}\right.$ and $\mathrm{s}, \mathrm{t} \in$ $\left.\operatorname{Std}_{r}(\lambda)\right\}$ is a cellular basis of $H_{r}(R ; \boldsymbol{q})$, with respect to the involution $*$ and the partially ordered set $\left(\widehat{\mathfrak{S}}_{r}, \triangleq\right)$.

We let $x_{\mathrm{t}}^{\lambda}$ denote the basis element of the cell module $\Delta_{r}(\lambda)$ corresponding to $\mathrm{t} \in \operatorname{Std}_{r}(\lambda), x_{\mathrm{t}}^{\lambda}=x_{\lambda} T_{w(\mathrm{t})}+H_{r}^{\triangleright \lambda}$. If $1 \leqslant a \leqslant i$, define

$$
T_{a, i}=T_{a} T_{a+1} \cdots T_{i-1},
$$


and $T_{i, a}=T_{a, i}^{*}$. If $\lambda \vdash i-1$ and $\mu \vdash i$, with $\mu=\lambda \cup\left\{\left(j, \mu_{j}\right)\right\}$, let $a=\sum_{k=1}^{j} \mu_{k}$. Define

$$
d_{\lambda \rightarrow \mu}=T_{a, i} \quad \text { and } \quad u_{\lambda \rightarrow \mu}=T_{i, a} \sum_{k=0}^{\lambda_{j}} T_{a, a-k},
$$

Given a path $\mathrm{t} \in \operatorname{Std}_{r}(\nu)$,

$$
\varnothing=\mathrm{t}(0) \rightarrow \mathrm{t}(1) \rightarrow \mathrm{t}(2) \rightarrow \cdots \rightarrow \mathrm{t}(r-1) \rightarrow \mathrm{t}(r)=\nu,
$$

define

$$
d_{\mathrm{t}}=d_{\mathrm{t}(r-1) \rightarrow \mathrm{t}(r)} d_{\mathrm{t}(r-2) \rightarrow \mathrm{t}(r-1)} \cdots d_{\mathrm{t}(0) \rightarrow \mathrm{t}(1)} .
$$

Proposition $5.2([4])$.

(1) For $\mathrm{t} \in \operatorname{Std}_{r}(\nu)$, one has $d_{\mathrm{t}}=T_{w(\mathrm{t})}$.

(2) For $\lambda \rightarrow \mu$ in $\widehat{\mathfrak{S}}$, one has $x_{\mu} d_{\lambda \rightarrow \mu}=u_{\lambda \rightarrow \mu}^{*} x_{\lambda}$.

Thus the Murphy cellular basis is recovered from the ordered products of branching factors: $x_{\mathrm{st}}^{\lambda}=\left(d_{\mathrm{s}}\right)^{*} x_{\lambda} d_{\mathrm{t}}$. Moreover, Remark 2.13 regarding factorization of representatives of the Murphy basis of cell modules applies to the Hecke algebra. We want to stress that Proposition 5.2 is established by computation and does not rely on the connection, established in [4], between the branching factors $d_{\lambda \rightarrow \mu}$ and $u_{\lambda \rightarrow \mu}$ and cell filtrations of restricted and induced cell modules.

Next, we recall the Jucys-Murphy elements for the Hecke algebras, see [11], Section 3.3 and Exercise 6, page 49. The JM elements in $H_{n}(\boldsymbol{q})$ are defined by

$$
L_{1}=1 \text { and } L_{k}=\boldsymbol{q}^{1-k} T_{k-1} \cdots T_{1} T_{1} \cdots T_{k-1} \text { for } k>1 .
$$

\section{Proposition 5.3.}

(1) $L_{1} L_{2} \cdots L_{r}$ is in the center of $H_{r}(\boldsymbol{q})$.

(2) The elements $L_{k}$ are multiplicative JM elements in the sense of Definition 4.3.

See [11, Section 3.3] for the proof. Again, the proof is computational and does not depend on deeper results on the Hecke algebras. We will now list several properties of the Hecke algebras and afterwards sketch two logical routes through this material, both using the ideas of Sections 3 and 4 .

(H1) The Hecke algebras $H_{r}(\mathbb{F}, \boldsymbol{q})$ are split semisimple.

(H2) The branching diagram for the sequence $\left(H_{r}(\mathbb{F}, \boldsymbol{q})\right)_{r \geqslant 0}$ of split semisimple algebras is Young's lattice.

(H3) The sequence of Hecke algebras over $R$ is restriction coherent, with $\Delta_{r-1}^{R}(\lambda)$ appearing as a subquotient of $\operatorname{Res}_{H_{r-1}(\boldsymbol{q})}^{H_{r}(\boldsymbol{q})}\left(\Delta_{r}^{R}(\mu)\right)$ if and only if $\lambda \subset \mu$.

(H4) More precisely, statement (3) of Theorem 2.9 holds.

Since the sequence $\left(H_{r}(\mathbb{F}, \boldsymbol{q})\right)_{r \geqslant 0}$ is a multiplicity free sequence of split semisimple algebras over $\mathbb{F}$, one can define Gelfand-Zeitlin idempotents as in Section 3.1 and seminormal bases as in Definition 3.2 using the GelfandZeitlin idempotents and the Murphy basis. 
(H5) The set $\left\{f_{\mathrm{t}}^{\lambda} \mid \mathrm{t} \in \operatorname{Std}_{r}(\lambda)\right\}$ is a basis of $\Delta_{r}^{\mathbb{F}}(\lambda)$ and the transition matrix between this basis and the Murphy basis is dominance unitriangular.

(H6) The JM elements act diagonally on the seminormal basis, $f_{\mathrm{t}}^{\lambda} L_{k}=$ $\boldsymbol{q}^{c_{\mathrm{t}}(k)} f_{\mathrm{t}}^{\lambda}$.

(H7) The JM elements act triangularly on the Murphy basis,

$$
x_{\mathrm{t}}^{\lambda} L_{k}=\boldsymbol{q}^{c_{\mathrm{t}}(k)} x_{\mathrm{t}}^{\lambda}+\sum_{\mathrm{s} \triangleright \mathrm{t}} r_{\mathrm{s}} x_{\mathrm{s}}^{\lambda} .
$$

We now sketch two logical paths through statements (H1)-(H7). Of course, there are many logical arrangements of this material, and these are just two possibilities.

First path. One can first establish that the Hecke algebras $H_{r}(\mathbb{F}, \boldsymbol{q})$ are split semisimple. One easy way to do this is as follows. Let $\langle\cdot, \cdot\rangle$ denote the bilinear form on each cell module arising from the Murphy basis. Let $\phi_{\lambda}$ be the determinant of the Gram matrix $\left[\left\langle m_{\mathrm{s}}^{\lambda}, m_{\mathrm{t}}^{\lambda}\right\rangle\right]_{\mathrm{s}, \mathrm{t}}$. Then $\phi_{\lambda} \in R=\mathbb{Z}\left[\boldsymbol{q}, \boldsymbol{q}^{-1}\right]$. Since the specialization of the Hecke algebra at $\boldsymbol{q}=1$ is the symmetric group algebra over $\mathbb{Q}$, which is semisimple, it follows that $\phi_{\lambda}(1) \neq 0$ and hence $\phi_{\lambda} \neq 0$. Now it follows from the general theory of cellular algebras that $H_{r}(\mathbb{F}, \boldsymbol{q})$ is split semisimple (see [11, Corollary 2.21]).

The next step, which is more substantial, is to show that (H3) holds. This is proved in [8] or [13]. This implies that (H2) holds as well, using [6, Lemma 2.2], which is elementary.

Taking into account Proposition 5.2, we now have verified axioms (D1) through (D6) for the Hecke algebras, as well as statements (1) and (2) of Theorem 2.9. A subtle point here is that we have not verified and do not need to verify at this point that the $d$-branching coefficients actually arise from the cell filtrations of restricted cell modules, i.e. that statement (3) of Theorem 2.9 holds.

We are now entitled to plug into the arguments of Sections 3 and 4, which give us the conclusions (H5) through (H7), but without revealing that the eigenvalues of the JM elements are $\boldsymbol{q}^{c_{\mathrm{t}}(k)}$. This additional information must come from an additional analysis, for example by extending the analysis of Okounkov and Vershik [16] to the Hecke algebras. Moreover, we obtain (H4) from Corollary 3.12.

Second path. The second path follows the analysis in [11, Chapter 3] and its abstraction in [12]. Start with [11, Theorem 3.32], which proves (H7). This implies that the JM elements $L_{k}$ are Jucys-Murphy elements in the sense of [12] and moreover satisfy the "separating condition" of [12, Section 3] over $\mathbb{F}$. One can therefore define idempotents $F_{\mathrm{t}}$ indexed by standard tableaux using interpolation formulas involving the JM elements as in [11, Section $3.3]$ or [12, Section 3], and the seminormal basis of the cell modules by $f_{\mathrm{t}}^{\lambda}=x_{\mathrm{t}}^{\lambda} F_{\mathrm{t}}$. From the general theory in [12, Section 3] one obtains (H5) and (H6). Moreover, the separating condition also implies (H1). The restriction rule $(\mathrm{H} 2)$ results from actually computing the seminormal representations 
as in [11, Theorem 3.36]. We are now left with the task of verifying (H3) and (H4).

Note that we have (D1) -(D4) as well as (D6) at our disposal as well as statements (1) and (2) of Theorem 2.9. Moreover, by [7, Proposition 3.11] the idempotents $F_{\mathrm{t}}$ obtained from the JM elements have to coincide with the Gelfand-Zeitlin idempotents. This is all we need to follow through the arguments of Section 3, and we end up with Corollary 3.12, which implies (H3) and (H4).

Remark 5.4. The net result of this discussion is that if one uses the result from the literature on the restriction coherence of the tower of Hecke algebras, one can avoid some of the work involved with showing dominance triangularity of the JM elements; and on the other hand, if one uses the results on dominance triangularity, one can avoid some of the work involved in proving restriction coherence (which was only recently proven in $[8,13]$ ).

\section{Skew CELl MOdUles FOR DiAGRAM ALGEBRAS}

In this section, we construct skew cell modules for diagram algebras and provide integral bases of these modules indexed by skew tableaux. We begin by constructing skew cell modules for $A_{r} \cap A_{s}^{\prime}$ when $0 \leqslant s \leqslant r$, and afterwards introduce a final axiom which allow us to view these modules as $A_{r-s}$ modules.

Let $0 \leqslant s<r, \nu \in \widehat{A}_{r}$, and $\lambda \in \widehat{A}_{s}$. Let $\mathrm{t}^{\lambda} \in \operatorname{Std}_{s}(\lambda)$ be maximal in $\operatorname{Std}_{s}(\lambda)$ with respect to the dominance order on paths. Define

$$
\Delta(\nu ; \triangleright \lambda)=\operatorname{Span}_{R}\left\{m_{\mathrm{t}}^{\nu} \mid \mathrm{t} \in \operatorname{Std}_{r}(\nu) \text { and } \mathrm{t}(s) \triangleright \lambda\right\},
$$

and

$$
\Delta\left(\nu ; \mathrm{t}^{\lambda}\right)=\operatorname{Span}_{R}\left\{m_{\mathrm{t}^{\lambda} \mathrm{ot}}^{\nu} \mid \mathrm{t} \in \operatorname{Std}_{r-s}(\nu \backslash \lambda)\right\},
$$

both $R$-submodules of $\Delta_{r}^{R}(\nu)$.

Lemma 6.1. $\Delta(\nu ; \triangleright \lambda)$ and $\Delta\left(\nu ; \mathrm{t}^{\lambda}\right)+\Delta(\nu ; \triangleright \lambda)$ are $A_{r} \cap A_{s}^{\prime}$ submodules of $\Delta_{r}^{R}(\nu)$.

Proof. Follows from Proposition 3.14 and maximality of $\mathrm{t}^{\lambda}$.

Let $\Delta(\nu \backslash \lambda)$ denote the $A_{r} \cap A_{s}^{\prime}$ module

$$
\Delta(\nu \backslash \lambda)=\left(\Delta\left(\nu ; \mathrm{t}^{\lambda}\right)+\Delta(\nu ; \triangleright \lambda)\right) / \Delta(\nu ; \triangleright \lambda) .
$$

\section{Lemma 6.2.}

(1) $\Delta(\nu \backslash \lambda)$ is, up to isomorphism, independent of the choice of the maximal element $\mathrm{t}^{\lambda}$.

(2) $\Delta\left(\nu ; \mathrm{t}^{\lambda}\right) F_{\mathrm{t}^{\lambda}} \subseteq \Delta_{r}^{\mathbb{F}}(\nu)$ is an $A_{r} \cap A_{s}^{\prime}$ submodule, and $\Delta(\nu \backslash \lambda) \cong \Delta\left(\nu ; \mathrm{t}^{\lambda}\right) F_{\mathrm{t}^{\lambda}}$.

(3) For any choice of $\mathbf{s} \in \operatorname{Std}_{s}(\lambda)$,

$$
\Delta^{\mathbb{F}}(\nu \backslash \lambda):=\Delta(\nu \backslash \lambda) \otimes_{R} \mathbb{F} \cong \Delta_{r}^{\mathbb{F}}(\nu) F_{\mathrm{s}}
$$

as $A_{r}^{\mathbb{F}} \cap\left(A_{s}^{\mathbb{F}}\right)^{\prime}$-modules. 
Proof. The first statement in part (2) follows from Lemma 6.1, because $\Delta(\nu ; \triangleright \lambda) F_{\mathrm{t}^{\lambda}}=0$. Now we have an $A_{r} \cap A_{s}^{\prime}$ module homomorphism $m \mapsto$ $m F_{\mathrm{t}^{\lambda}}$ from $\Delta\left(\nu ; \mathrm{t}^{\lambda}\right)+\Delta(\nu ; \triangleright \lambda)$ to $\Delta\left(\nu ; \mathrm{t}^{\lambda}\right) F_{\mathrm{t}^{\lambda}}$ with kernel $\Delta(\nu ; \triangleright \lambda)$, which gives the isomorphism in part (2). If $\mathrm{t}_{1}$ and $\mathrm{t}_{2}$ are two maximal elements in $\operatorname{Std}_{s}(\lambda)$, then right multiplication by $E_{\mathrm{t}_{1} \mathrm{t}_{2}}^{\lambda}$ is an $A_{r} \cap A_{s}^{\prime}$-module isomorphism from $\Delta\left(\nu ; \mathrm{t}_{1}\right) F_{\mathrm{t}_{1}}$ to $\Delta\left(\nu ; \mathrm{t}_{2}\right) F_{\mathrm{t}_{2}}$, which proves part (1).

For part (3), using Theorem 3.5 and Proposition 3.9 part (3), first note that given any $\mathrm{s}, \overline{\mathrm{s}} \in \operatorname{Std}_{s}(\lambda)$, we have that $\Delta_{r}^{\mathbb{F}}(\nu) F_{\mathrm{s}} \cong \Delta_{r}^{\mathbb{F}}(\nu) F_{\overline{\mathrm{s}}}$ as $R-$ modules, with the isomorphism realized by right multiplication by $E_{\mathrm{s} s}^{\lambda}$. This map preserves the $A_{r}^{\mathbb{F}} \cap\left(A_{s}^{\mathbb{F}}\right)^{\prime}$-module structure, since $A_{r}^{\mathbb{F}} \cap\left(A_{s}^{\mathbb{F}}\right)^{\prime}$ commutes with $E_{\mathrm{s} \bar{s}}^{\lambda}$. In particular, we can set $\mathrm{s}=\mathrm{t}^{\lambda}$ without loss of generality. Now, it follows from Theorem 3.5 that

$$
\Delta^{\mathbb{F}}(\nu ; \triangleright \lambda):=\Delta(\nu ; \triangleright \lambda) \otimes_{R} \mathbb{F}=\operatorname{Span}_{\mathbb{F}}\left\{f_{\mathrm{t}}^{\nu} \mid \mathrm{t} \in \operatorname{Std}_{r}(\nu) \text { and } \mathrm{t}(s) \triangleright \lambda\right\},
$$

and

$$
\Delta\left(\nu ; \mathrm{t}^{\lambda}\right) \otimes_{R} \mathbb{F}+\Delta^{\mathbb{F}}(\nu ; \triangleright \lambda)=\operatorname{Span}_{\mathbb{F}}\left\{f_{\mathrm{t}^{\lambda} \mathrm{ot}}^{\nu} \mid \mathrm{t} \in \operatorname{Std}_{r-s}(\nu \backslash \lambda)\right\}+\Delta^{\mathbb{F}}(\nu ; \triangleright \lambda) .
$$

Arguing as for part (2), we have

$$
\Delta^{\mathbb{F}}(\nu \backslash \lambda) \cong \operatorname{Span}_{\mathbb{F}}\left\{f_{\mathrm{t}^{\lambda} \text { ot }}^{\nu} \mid \mathrm{t} \in \operatorname{Std}_{r-s}(\nu \backslash \lambda)\right\} F_{\mathrm{t}^{\lambda}} .
$$

Now one easily verifies that $\operatorname{Span}_{\mathbb{F}}\left\{f_{\mathrm{t}^{\lambda} \circ \mathrm{t}}^{\nu} \mid \mathrm{t} \in \operatorname{Std}_{r-s}(\nu \backslash \lambda)\right\} F_{\mathrm{t}^{\lambda}}=\Delta_{r}^{\mathbb{F}}(\nu) F_{\mathrm{t}^{\lambda}}$.

Now we introduce one final axiom for towers of diagram algebras, which allows us to regard $\Delta(\nu \backslash \lambda)$ as an $A_{r-s}$-module.

(D7) There is a an automorphism, $f_{r}$, of order 2 of each $A_{r}$ such that for each $s$ with $0 \leqslant s \leqslant r, f_{r}\left(A_{r-s}\right)$ commutes with $A_{s} \subset A_{r}$.

Using this final axiom, we can define a homomorphism $\varphi_{r, s}: A_{s} \otimes_{R} A_{r-s} \rightarrow$ $A_{r}$ by

$$
\varphi_{r, s}(a \otimes b)=a f_{r}\left(f_{r-s}(b)\right),
$$

where both $A_{s}$ and $A_{r-s}$ are regarded as subalgebras of $A_{r}$ via the usual embeddings. We can restrict any $A_{r}$-module to $A_{s} \otimes_{R} A_{r-s}$, by composing with $\varphi_{r, s}$.

Remark 6.3. Condition (D7) is satisfied by the group algebras of symmetric groups, the Hecke algebras, and the Brauer, BMW, partition, and Temperley-Lieb algebras. For each of these examples, the involution $f_{r}$ is given by flipping a diagram or tangle through its vertical axis (whereas * is given by flipping a diagram through its horizontal axis). Also, in each of these genuine diagram or tangle algebras, one can define a tensor product operation, i.e. a homomorphism from $A_{s} \otimes_{R} A_{r-s}$ to $A_{r}$, which, on the level of diagrams or tangles, is just placing diagrams side by side. Taking $f_{r}$ to be the flip through a vertical axis, the homomorphism $\varphi_{r, s}$ defined above agrees with the homomorphism determined by placing diagrams side by side. Note that $f_{r} \circ f_{r-s}$ is the shift operation on $A_{r-s}$ determined on the level of diagrams by adding $s$ vertical strands to the left of a diagram in $A_{r-s}$. 
Denote by $\Delta_{r-s}(\nu \backslash \lambda)$ the $A_{r} \cap A_{s}^{\prime}$ module $\Delta(\nu \backslash \lambda)$, when regarded as an $A_{r-s}$-module by composing with the homomorphism $f_{r} \circ f_{r-s}: A_{r-s} \rightarrow$ $A_{r} \cap A_{s}^{\prime}$.

Definition 6.4. Let $\left(A_{r}\right)_{r \geqslant 0}$ denote a tower of algebras satisfying conditions (D1) - (D7). Given $\lambda \in \widehat{A}_{s}$ and $\nu \in \widehat{A}_{r}$, the $A_{r-s}$-module $\Delta_{r-s}^{R}(\nu \backslash \lambda)$ described above is called the skew cell module associated to $\lambda$ and $\nu$.

Remark 6.5. The definition of skew cell modules generalizes the definition of skew Specht modules for the tower of symmetric group algebras $\left(\mathbb{Z} \mathfrak{S}_{r}\right)_{r \geqslant 0}$. The skew Specht module $S^{\nu \backslash \lambda}$ for a skew shape $\nu \backslash \lambda$ is defined as the span of polytabloids of shape $\nu \backslash \lambda$; see [10, Section 4] for the definition of polytabloids, and [19] for the definition of skew Specht modules. Let us regard $S^{\nu \backslash \lambda}$ as an $\mathfrak{S}_{\{s+1, \ldots, r\}}$-module, where $|\lambda|=s$ and $|\nu|=r$. Let $\mathrm{t}_{\lambda}$ be the column reading standard tableau of shape $\lambda$, so $t_{\lambda}$ is the unique maximal tableau in column dominance order $\unrhd_{\text {col }}$ on standard tableaux of shape $\lambda$. Consider the following subsets of the Specht module $S^{\nu}$ :

$$
S\left(\nu ; \triangleright_{\text {col }} \lambda\right)=\operatorname{Span}_{\mathbb{Z}}\left\{e_{\mathrm{t}} \mid \mathrm{t} \in \operatorname{Std}_{r}(\nu) \text { and } \mathrm{t}(s) \triangleright_{\text {col }} \lambda\right\}
$$

and

$$
S\left(\nu ; \mathrm{t}_{\lambda}\right)=\operatorname{Span}_{\mathbb{Z}}\left\{e_{\mathrm{t}_{\lambda} \text { os }} \mid \mathrm{s} \in \operatorname{Std}_{r-s}(\nu \backslash \lambda)\right\},
$$

where $e_{\mathrm{t}}$ denotes a polytabloid. Using the Garnir relations [10], one can verify that $S\left(\nu ; \triangleright_{\mathrm{col}} \lambda\right)$ and $S\left(\nu ; \mathrm{t}_{\lambda}\right)+S\left(\nu ; \triangleright_{\mathrm{col}} \lambda\right)$ are $\mathfrak{S}_{\{s+1, \ldots, r\}}$ submodules of $S^{\nu}$, and the quotient of these modules is isomorphic to the skew cell module $S^{\nu \backslash \lambda}$. A more general statement is proved in [19, Theorem 3.1]. Modulo the identification of cell modules of $\mathfrak{S}_{r}$ with classical Specht modules - which involves both transpose of diagrams and twisting by the automorphism $s_{i} \mapsto-s_{i}$, see [15, Section 5] - this shows that the classical skew Specht modules agree with the skew cell modules defined here.

Proposition 6.6. Given $\lambda \in \widehat{A}_{s}, \nu \in \widehat{A}_{r}$, and an $A_{r-s}^{\mathbb{F}}-$ module $M$, we have

$\operatorname{Hom}_{A_{s}^{\mathbb{F}} \otimes A_{r-s}^{\mathbb{F}}}\left(\Delta_{s}^{\mathbb{F}}(\lambda) \otimes_{\mathbb{F}} M, \operatorname{Res}_{A_{s}^{\mathbb{F}} \otimes A_{r-s}^{\mathbb{F}}}^{A^{\mathbb{F}}}\left(\Delta_{r}^{\mathbb{F}}(\nu)\right) \cong \operatorname{Hom}_{A_{r-s}^{\mathbb{F}}}\left(M, \Delta_{r-s}^{\mathbb{F}}(\nu \backslash \lambda)\right)\right.$.

Proof. Identify $\Delta_{r-s}^{\mathbb{F}}(\nu \backslash \lambda)$ with $\Delta_{r}^{\mathbb{F}}(\nu) F_{\mathrm{t}^{\lambda}}$, using Lemma 6.2 part (2). For $\varphi \in \operatorname{Hom}_{A_{s}^{\mathbb{F}} \otimes A_{r-s}^{\mathbb{F}}}\left(\Delta_{s}^{\mathbb{F}}(\lambda) \otimes_{\mathbb{F}} M, \operatorname{Res}_{A_{s}^{\mathbb{F}} \otimes A_{r-s}^{\mathbb{F}}}^{A_{\mathbb{F}}^{\mathbb{F}}}\left(\Delta_{r}^{\mathbb{F}}(\nu)\right)\right.$, define $\bar{\varphi}$ by

$$
\bar{\varphi}(m)=\varphi\left(f_{\mathrm{t}^{\lambda}}^{\lambda} \otimes m\right)=\varphi\left(f_{\mathrm{t}^{\lambda}}^{\lambda} F_{\mathrm{t}^{\lambda}} \otimes m\right)=\varphi\left(f_{\mathrm{t}^{\lambda}}^{\lambda} \otimes m\right) F_{\mathrm{t}^{\lambda}} .
$$

It follows that $\bar{\varphi} \in \operatorname{Hom}_{A_{r-s}^{\mathbb{F}}}\left(M, \Delta_{r-s}^{\mathbb{F}}(\nu \backslash \lambda)\right)$. We have to check that the $\operatorname{map} \varphi \mapsto \bar{\varphi}$ is an isomorphism. For injectivity, suppose $\bar{\varphi}=0$. Then for all $m \in M$ and all $x \in A_{s}^{\mathbb{F}}, \varphi\left(f_{\mathrm{t}^{\lambda}}^{\lambda} x \otimes m\right)=\bar{\varphi}(m) x=0$. Hence $\varphi=0$ since $f_{\mathrm{t}^{\lambda}}^{\lambda} A_{s}^{\mathbb{F}}=\Delta_{s}^{\mathbb{F}}(\lambda)$. For surjectivity, let $\psi \in \operatorname{Hom}_{A_{r-s}^{\mathbb{F}}}\left(M, \Delta_{r}^{\mathbb{F}}(\nu) F_{\mathrm{t}^{\lambda}}\right)$. Define $\varphi$ by $\varphi\left(f_{\mathrm{t}^{\lambda}}^{\lambda} x \otimes m\right)=\psi(m) x=\psi(m) F_{\mathrm{t}^{\lambda}} x$ for $x \in A_{s}^{\mathbb{F}}$. Then $\varphi$ is well-defined because $f_{\mathrm{t}^{\lambda}}^{\lambda} x=0 \Leftrightarrow F_{\mathrm{t}^{\lambda}} x=0$. Now one can easily check that $\varphi$ is an $A_{s}^{\mathbb{F}} \otimes A_{r-s}^{\mathbb{F}}$-homomorphism and that $\bar{\varphi}=\psi$. 
Finally, let $\left(A_{r}\right)_{r \geqslant 0}$ denote a tower of algebras satisfying (D1)-(D7). Let $\lambda \in \widehat{A}_{s}, \nu \in \widehat{A}_{r}, \mu \in \widehat{A}_{r-s}$ and define associated multiplicities

$$
\begin{aligned}
& A_{\lambda, \mu}^{\nu}=\operatorname{dim}_{\mathbb{F}} \operatorname{Hom}_{A_{r-s}^{\mathbb{F}}}\left(m_{\mu} A_{r-s}^{\mathbb{F}}, \Delta_{r-s}^{\mathbb{F}}(\nu \backslash \lambda)\right) \\
& a_{\lambda, \mu}^{\nu}=\operatorname{dim}_{\mathbb{F}} \operatorname{Hom}_{A_{r-s}^{\mathbb{F}}}\left(\Delta_{r-s}^{\mathbb{F}}(\mu), \Delta_{r-s}^{\mathbb{F}}(\nu \backslash \lambda)\right) .
\end{aligned}
$$

We recall that $\left(m_{\mu} A_{r-s} /\left(m_{\mu} A_{r-s} \cap A_{r-s}^{\triangleright \mu}\right)\right.$ is isomorphic to the cell module $\Delta_{s}(\mu)$ and therefore $a_{\lambda, \mu}^{\nu} \leqslant A_{\lambda, \mu}^{\nu}$, by definition.

Remark 6.7. Consider the tower of the group algebras of symmetric groups $\left(\mathfrak{S}_{r}\right)_{r \geqslant 0}$. Recall that a partition $\mu$ is defined to be a finite weakly decreasing sequence of non-negative integers. We define the degree of the partition $\lambda$ to be the sum, $|\mu|$, over all non-zero terms in this sequence. Recall that $\widehat{\mathfrak{S}}_{r-s}$ is the set of partitions of degree $r-s$, see [4]. Given $\mu$ a partition of $r-s$, the module $m_{\mu} \mathfrak{S}_{r-s}^{\mathbb{F}}$ is isomorphic to the so-called Young permutation module, that is the module obtained by induction from the subgroup $\mathfrak{S}_{\mu}$ which stabilizes the set $\left\{1, \ldots, \mu_{1}\right\} \times\left\{\mu_{1}+1, \ldots, \mu_{1}+\mu_{2}\right\} \times \cdots \subseteq\{1, \ldots, r-$ $s\}$. Therefore, the coefficients defined in (11) and (12) above are the skewKostka and Littlewood-Richardson coefficients, respectively [20, Pages 311 and 338].

6.1. The stable Kronecker coefficients. Given $\lambda=\left(\lambda_{1}, \lambda_{2}, \ldots, \lambda_{\ell}\right)$ a partition and $n \in \mathbb{N}$ sufficiently large we set

$$
\lambda_{[n]}=\left(n-|\lambda|, \lambda_{1}, \lambda_{2}, \ldots, \lambda_{\ell}\right) .
$$

Given $n \in \mathbb{N}$, we recall that $\mathfrak{S}_{n}$ denotes the symmetric group on $n$ letters. The tower of algebras $\left(\mathbb{Z} \mathfrak{S}_{n}\right)_{n \geqslant 0}$ satisfy conditions (D1)-(D7). Given $r \in$ $\frac{1}{2} \mathbb{N}$, we let $P_{2 r}(n)$ denote the partition algebra on $r$ strands with parameter $n \in \mathbb{N}$. The tower of algebras $\left(P_{r}(n)\right)_{r \geqslant 0}$ satisfy conditions (D1)-(D7). The representation theories of the symmetric groups and partition algebras are intimately related via a generalizations of classical Schur-Weyl duality. Through this duality, we obtain the following theorem.

Theorem 6.8. Let $\lambda$ be a partition of degree $s, \mu$ be a partition of degree $r-s$, and $\nu$ be a partition of degree less than or equal to $r$. We have that

$$
\begin{aligned}
& \operatorname{Hom}_{\mathbb{Q S}_{n}}\left(\Delta_{\mathbb{Q S}_{n}}\left(\lambda_{[n]}\right) \otimes \Delta_{\mathbb{Q S}_{n}}\left(\mu_{[n]}\right), \Delta_{\mathbb{Q S}_{n}}\left(\nu_{[n]}\right)\right) \\
\cong & \operatorname{Hom}_{P_{r-s}^{\mathbb{Q}}(n)}\left(\Delta_{P_{r-s}^{\mathbb{Q}}(n)}(\mu), \Delta_{P_{r-s}^{\mathbb{Q}}(n)}(\nu \backslash \lambda)\right) .
\end{aligned}
$$

for $n$ sufficiently large $(n \geqslant 2 r$ will suffice).

Proof. This follows immediately from Proposition 6.6 and [2, Corollary 3.4].

Given $\lambda \vdash r-s, \mu \vdash s$ and $\nu \vdash r$ and $n \geqslant 2 r$ we are interested in the multiplicities

$$
\begin{gathered}
P_{\lambda, \mu}^{\nu}=\operatorname{dim}_{\mathbb{Q}} \operatorname{Hom}_{P_{r-s}^{\mathbb{Q}}(n)}\left(m_{\mu} P_{r-s}^{\mathbb{Q}}(n), \Delta_{r-s}^{\mathbb{Q}}(\nu \backslash \lambda)\right) \\
p_{\lambda, \mu}^{\nu}=\operatorname{dim}_{\mathbb{Q}} \operatorname{Hom}_{P_{r-s}^{\mathbb{Q}}(n)}\left(\Delta_{r-s}^{\mathbb{Q}}(\mu), \Delta_{r-s}^{\mathbb{Q}}(\nu \backslash \lambda)\right)
\end{gathered}
$$


for a cell quasi-idempotent $m_{\mu} \in P_{r-s}^{\mathbb{Q}}(n)$. By Theorem 6.8 and [2], the coefficients $p_{\lambda, \mu}^{\nu}$ are equal to the stable Kronecker coefficients. These coefficients have been described as "perhaps the most challenging, deep and mysterious objects in algebraic combinatorics' [18]. On the other hand, the coefficients $P_{\lambda, \mu}^{\nu}$ do not seem to have been studied anywhere in the literature. Motivated by the classical case, we ask the following questions:

- can one interpret the coefficients $P_{\lambda, \mu}^{\nu}$ and $p_{\lambda, \mu}^{\nu}$ in terms of the combinatorics of skew-tableaux for the partition algebra?

- do there exist natural generalizations of the semistandard and lattice permutation conditions in this setting?

- do the coefficients $P_{\lambda, \mu}^{\nu}$ provide a first step towards understanding the stable Kronecker coefficients $p_{\lambda, \mu}^{\nu}$ ?

The first two authors shall address these questions in an upcoming series of papers with Maud De Visscher. In particular, we use the above interpretation to provide a positive combinatorial description of the stable Kronecker coefficients labelled by an infinite family of triples of partitions (including the Littlewood-Richardson coefficients, and Kronecker coefficients indexed by two two-row partitions as important examples).

Acknowledgements. We would like to thank the Royal Commission for the Exhibition of 1851 and EPSRC grant EP/L01078X/1 for financial support.

\section{REFERENCES}

1. C. Bowman, M. De Visscher, and J. Enyang, Simple modules for the partition algebra and stability of Kronecker coefficients, in preparation (2015).

2. C. Bowman, M. De Visscher, and R. Orellana, The partition algebra and the Kronecker coefficients, Trans. Amer. Math. Soc. 367 (2015), no. 5, 3647-3667. MR 3314819

3. C. Bowman, J. Enyang, and F. Goodman, The cellular second fundamental theorem of invariant theory for classical groups, preprint (2016), arXiv 1610.0900.

4. John Enyang and F. M. Goodman, Cellular Bases for Algebras with a Jones Basic Construction, Algebr. Represent. Theory 20 (2017), no. 1, 71-121. MR 3606482

5. T. Geetha and F. M. Goodman, Cellularity of wreath product algebras and A-Brauer algebras, J. Algebra 389 (2013), 151-190. MR 3065998

6. F. M. Goodman and J. Graber, Cellularity and the Jones basic construction, Adv. in Appl. Math. 46 (2011), no. 1-4, 312-362. MR 2794027

7. _ On cellular algebras with Jucys Murphy elements, J. Algebra 330 (2011), 147-176. MR 2774622

8. F. M. Goodman, Ross Kilgore, and Nicholas Teff, A cell filtration of the restriction of a cell module, preprint (2015), arXiv 1504.02136.

9. J. J. Graham and G. I. Lehrer, Cellular algebras, Invent. Math. 123 (1996), no. 1, 1-34. MR 1376244

10. G. D. James, The representation theory of the symmetric groups, Lecture Notes in Mathematics, vol. 682, Springer, Berlin, 1978. MR 513828

11. A. Mathas, Iwahori-Hecke algebras and Schur algebras of the symmetric group, University Lecture Series, vol. 15, American Mathematical Society, Providence, RI, 1999. MR 1711316

12. __ Seminormal forms and Gram determinants for cellular algebras, J. Reine Angew. Math. 619 (2008), 141-173, With an appendix by Marcos Soriano. MR 2414949

13. A. Mathas, Restricting Specht modules of cyclotomic Hecke algebras, Science China Mathematics (to appear). 
14. G. E. Murphy, On the representation theory of the symmetric groups and associated Hecke algebras, J. Algebra 152 (1992), no. 2, 492-513. MR 1194316

15. - The representations of Hecke algebras of type $A_{n}$, J. Algebra 173 (1995), no. 1, 97-121. MR 1327362

16. A. Okounkov and A. Vershik, A new approach to representation theory of symmetric groups, Selecta Math. (N.S.) 2 (1996), no. 4, 581-605. MR 1443185 (99g:20024)

17. _ A new approach to the representation theory of the symmetric groups. II, Journal of Mathematical Sciences 131 (2005), 5471-5494.

18. Igor Pak and Greta Panova, Bounds on certain classes of Kronecker and q-binomial coefficients, J. Combin. Theory Ser. A 147 (2017), 1-17. MR 3589885

19. M. H. Peel and G. D. James, Specht series for skew representations of symmetric groups, J. Algebra 56 (1979), no. 2, 343-364. MR 528580

20. Richard P. Stanley, Enumerative combinatorics. Vol. 2, Cambridge Studies in Advanced Mathematics, vol. 62, Cambridge University Press, Cambridge, 1999, With a foreword by Gian-Carlo Rota and appendix 1 by Sergey Fomin. MR 1676282 